\title{
Differential modular forms on Shimura curves, II: Serre operators
}

\author{
Alexandru Buium
}

\begin{abstract}
One of the main results announced in Part I (Compositio Math. 139 (2003), 197-237) is proved. The main technique consists in developing a coordinate free version of some of the theory of Serre operators on differential modular forms introduced by Barcau.
\end{abstract}

\section{Introduction, main concepts, and main results}

\subsection{Introduction}

This paper is a direct continuation of [Bui03]; among other things we will prove here the last assertion of Theorem 1.2 in [Bui03] which was stated without proof in [Bui03]. For the convenience of the reader, the Introduction to the present paper will be made independent of [Bui03]; on the other hand, in the main body of the paper, we will rely heavily on the concepts and results of [Bui03].

Differential modular forms were introduced in [Bui00]. Our main motivation was to provide a geometric setting for the quotient of a modular curve by the isogeny equivalence relation. This quotient does not exist in usual algebraic geometry but, rather, in an extension of the latter; this extension of algebraic geometry can be called $\delta$-algebraic geometry and was introduced in [Bui95] and [Bui96]. In [Bar03], Barcau introduced a new technique in the study of differential modular forms. His technique was based on an analogue, in $\delta$-algebraic geometry, of Serre's operators on classical modular forms [Kat73b]. Some of the main results in [Bar03] depend however on the explicit structure of the algebra of classical modular forms and involve computations in the "coordinates given by the Eisenstein series $E_{4}$ and $E_{6}$ '. One of the aims of the present paper is to develop a 'coordinate free' approach to some of the theory in [Bar03]. This allows us to apply the technique of Serre operators to differential modular forms in situations where 'no coordinates are available' (e.g. for modular curves with level structures or for Shimura curves); for instance we will prove, in this way, the last assertion of Theorem 1.2 in [Bui03].

Throughout this paper we denote by $W:=\mathbb{Z}[\phi]$ the ring of polynomials with $\mathbb{Z}$-coefficients in a variable $\phi$. For $w=w(\phi)=\sum a_{i} \phi^{i} \in W$ and $s \in \mathbb{Z}$ we set $w(s)=\sum a_{i} s^{i}$. We set $\operatorname{deg}(w):=$ $\sum a_{i}=w(1)$. (So deg here is not the degree of $w$ as a polynomial in $\phi$ but rather as an element in the semigroup ring of the semigroup $\mathbb{Z}_{+}$.) We let ord $(w)$ be the largest integer $i$ such that $a_{i} \neq 0$; if $w=0$ we set $\operatorname{ord}(w)=0$. We denote by $\operatorname{ord}(\bar{w})$ the largest integer $i$ such that $a_{i} \not \equiv 0 \bmod p$; we set $\operatorname{ord}(\bar{w})=0$ if $w \in p W$. We let $W_{+}$be the set of all $w \in W$ with $a_{i} \geqslant 0$ for all $i$. We let $W(r)$ be the subgroup of all $w \in W$ such that $\operatorname{ord}(w) \leqslant r$. Let $\phi$ act as a ring endomorphism of a ring $A$. Assume that either $\lambda \in A^{\times}, w \in W$, or that $\lambda \in A, w \in W_{+}$; then we write

$$
\lambda^{w}:=\lambda^{a_{0}}(\phi(\lambda))^{a_{1}} \cdots\left(\phi^{m}(\lambda)\right)^{a_{m}} \in A .
$$

Received 17 August 2002, accepted in final form 24 April 2003. 2000 Mathematics Subject Classification 11G18, $14 \mathrm{G} 35$.

Keywords: Shimura curves, differential modular forms.

The author was supported in part by NSF Grant \#0096946.

This journal is (c) Foundation Compositio Mathematica 2004. 


\section{A. Buium}

Throughout the paper $R$ will denote the completion of the maximum unramified extension of the ring of $p$-adic integers, $\mathbb{Z}_{p}$, where $p$ is a prime $\geqslant 5$. We let $k=R / p R=\overline{\boldsymbol{F}}_{p}$, and we let $K$ be the quotient field of $R$. We let $\phi$ act on $R$ as the unique ring automorphism lifting the $p$-power Frobenius on $k$. The rest of this section is devoted to introducing our main objects and stating our main results. In $\S \S 2-7$ we develop the Serre operator theory (discussing the modular and Shimura cases simultaneously). Section 8 is devoted to the conclusion of the proofs of our main results.

\subsection{Shimura curves}

Let $D$ be a non-split indefinite quaternion algebra over the rationals equipped with an order $\mathcal{O}_{D}$ which is stable under the canonical involution. We assume $D$ is split at $p$. Let $X$ be a smooth affine curve over $R$ with geometrically irreducible fibers and let $(A, i, \theta)$ be a polarized false elliptic curve over $X$ which is formally universal at each $k$-point of $X$; here $i: \mathcal{O}_{D} \rightarrow \operatorname{End}(A)$ is the 'multiplication by $\mathcal{O}_{D}$ ' map, $\theta$ is the unique 'natural principal polarization compatible with $i$ ', and $A$ is said to be formally universal at a $k$-point of $X$ if the completion of $A$ along its fiber at that point is the formal universal deformation of that fiber (cf. [DT94], [Buz97], [Bui03]). For simplicity, we shall refer to $X$ as being a Shimura curve. (For instance $X$ can be an affine étale open set of the pull-back to $R$ of a Shimura curve over $\mathbb{Z}[1 / N], N \not \equiv 0 \bmod p$, parameterizing families of false elliptic curves with some level structure that makes the moduli problem representable, cf. [Buz97]; sometimes these curves are referred to as fake/false modular curves.) We fix now a $k$-point $P_{0} \in X(k)$ of $X$ and we let $\left(A_{0}, i_{0}, \theta_{0}\right)$ be the polarized false elliptic curve over $k$ obtained from $(A, i, \theta)$ by pull-back via $P_{0}$. We assume $A_{0}$ is ordinary and $\left(A_{0}, \theta_{0}\right)$ is a Jacobian. (By [Bui03, Lemma 2.6], for any fixed $D$, there exists $p_{0}$ such that for all primes $p \geqslant p_{0}$ there exists a Shimura curve $X$ containing a $k$-point that corresponds to an ordinary Jacobian.) We denote by $\hat{X}$ the $p$-adic completion of $X$ and by $X^{\text {for }}$ the completion of $X$ at the closed point $P_{0}$. We let $S=\mathcal{O}(\hat{X})$ and $S_{\text {for }}=\mathcal{O}\left(X^{\text {for }}\right)$. By abuse of notation we still denote by $(A, i, \theta)$ the induced false elliptic curve over $S$ or $S_{\text {for }}$ respectively. If, in addition, one is given bases $b$ and $\check{b}$ of the physical Tate modules $T_{p} A_{0}$ and $T_{p} \check{A}_{0}$ respectively such that $\left(A_{0}, i_{0}, \theta_{0}, b, \check{b}\right)$ is a $D$-frame (in the sense of [Bui03, $\left.\left.\S 2.4\right]\right)$ then we have a natural identification $S_{\text {for }} \simeq R[[T]]$. (Recall that $\left(A_{0}, i_{0}, \theta_{0}, b, \breve{b}\right)$ is called a $D$-frame if $b$ and $\breve{b}$ correspond to each other via the polarization $\theta_{0}: A_{0} \rightarrow \check{A}_{0}$ and, in addition, any lifting of $\left(A_{0}, i_{0}\right)$ to $R$ has Serre-Tate matrix, with respect to $b$ and $\breve{b}$, of the form $\operatorname{diag}\left(q, q^{d}\right), d:=\operatorname{disc}(D)$.) Throughout the paper, whenever we refer to the Shimura curve $X$ we assume that we have fixed the data:

$$
A, i, P_{0}, b, \check{b} \text {. }
$$

Let us note that $\mathcal{O}_{D}$ acts on the $S$-module $H^{10}:=H^{0}\left(A, \Omega_{A / S}^{1}\right)$. Let $e_{11}$ be the image of the idempotent $\left(\begin{array}{ll}1 & 0 \\ 0 & 0\end{array}\right)$ via the map

$$
\operatorname{Mat}_{2}(S)^{\mathrm{op}} \simeq \mathcal{O}_{D}^{\mathrm{op}} \otimes_{\mathbb{Z}} S \rightarrow \operatorname{End}_{S}\left(H^{10}\right)
$$

where the isomorphism above is induced by a fixed isomorphism

$$
j: \mathcal{O}_{D} \otimes \mathbb{Z}_{p} \simeq \operatorname{Mat}_{2}\left(\mathbb{Z}_{p}\right)
$$

as in [Bui03, $\S 1.2]$. Then define the $S$-module of false 1 -forms

$$
\mathcal{L}:=e_{11} H^{10} \text {. }
$$

Replacing $S$ by $S_{\text {for }}$ in the construction above we define in a similar way the $S_{\text {for-module }} \mathcal{L}_{\text {for }}$.

\subsection{Modular curves}

Similar objects can be considered in the modular curve context. If $X$ is a smooth curve over $R$, with geometrically irreducible fibers, and $A$ is a smooth elliptic curve over $X$ which is formally universal at each $k$-point of $X$, then we shall refer to $X$ as being a modular curve. (For instance 


\section{Differential MODUlar FORMS ON SHIMURA CURVES, II}

$X$ can be an étale affine open set of the pull-back to $R$ of a modular curve over $\mathbb{Z}[1 / N], N \not \equiv 0$ mod $p$, parameterizing families of elliptic curves with some level structure that makes the moduli problem representable.) We fix a $k$-point $P_{0} \in X(k)$ corresponding to an ordinary elliptic curve. We may consider the corresponding objects $S, S_{\text {for }}, A, A_{0}$, etc. We define the $S$-module of 1-forms, as usual, by $\mathcal{L}=H^{10}=H^{0}\left(A, \Omega_{A / S}\right)$. Replacing $S$ by $S_{\text {for }}$ in the construction above we define in a similar way the $S_{\text {for-module }} \mathcal{L}_{\text {for }}$. If $\theta: A \rightarrow \check{A}$ is the natural polarization and $\left(A_{0}, \theta_{0}, b, \check{b}\right)$ is a 1 -frame (in the sense of [Bui03, § 2]) then we have an induced identification $S_{\text {for }} \simeq R[[T]]$. (Recall that $\left(A_{0}, \theta_{0}, b, \breve{b}\right)$ is a 1 -frame if $b$ and $\breve{b}$ are bases of the physical Tate modules $T_{p} A_{0}$ and $T_{p} \check{A}_{0}$ respectively which correspond to each other via the polarization $\theta_{0}$.) Throughout the paper, whenever we refer to the modular curve $X$ we will assume we have fixed the data:

$$
A, P_{0}, b, \check{b} \text {. }
$$

\subsection{Differential modular forms on $X$ and $X^{\text {for }}$}

In what follows we assume $X$ is either a modular or a Shimura curve and we consider a certain sequence of rings $\left(S^{r}\right)$ and morphisms $\varphi, \phi: S^{r} \rightarrow S^{r+1}$ as follows. We define $S^{r}$ to be the ring of global sections

$$
S^{r}=\mathcal{O}\left(J^{r}(X)\right)
$$

on the $p$-jet space of order $r, J^{r}(\hat{X})=J^{r}(X)$ of $X / R$ in the sense of [Bui00, p. 103]. The morphisms $\varphi$ are induced by the natural projections $J^{r+1}(X) \rightarrow J^{r}(X)$ while the morphisms $\phi$ are defined by $\phi(x)=x^{p}+\delta x$ where $\delta$ is the natural ' $p$-derivation' in the theory of $p$-jet spaces of [Bui00, $\S 1$. The datum $S^{*}=\left(S^{r}, \varphi, \phi\right)$ defines a prolongation sequence in the sense of [Bui00, $\left.\S 1\right]$; for the purpose of this introduction we may ignore this. Also if $S_{\text {for }} \simeq R[[T]]$ corresponds to a given $D$-frame or 1-frame respectively, we consider a certain sequence of rings $\left(S_{\text {for }}^{r}\right)$ and morphisms $\varphi, \phi: S_{\text {for }}^{r} \rightarrow S_{\text {for }}^{r+1}$ as follows. We set

$$
S_{\text {for }}^{r}:=R[[T]]\left[T^{\prime}, \ldots, T^{(r)}\right]^{\wedge},
$$

where ${ }^{\wedge}$ denotes $p$-adic completion. The morphisms $\varphi$ are the natural inclusions. The morphisms $\phi$ are defined by $\phi(x)=x^{p}+\delta x$ where $\delta$ is the unique $p$-derivation with $\delta T=T^{\prime}, \delta T^{\prime}=T^{\prime \prime}$, and so on. Again we have defined a prolongation sequence $S_{\text {for }}^{*}$. For each $i \leqslant r$ we may consider the ring homomorphism

$$
\varphi^{r-i} \phi^{i}: S \stackrel{\phi}{\rightarrow} S^{1} \stackrel{\phi}{\rightarrow} S^{2} \stackrel{\phi}{\rightarrow} \cdots \stackrel{\phi}{\rightarrow} S^{i} \stackrel{\varphi}{\rightarrow} S^{i+1} \stackrel{\varphi}{\rightarrow} \cdots \stackrel{\varphi}{\rightarrow} S^{r}
$$

and we may define

$$
\mathcal{L}^{\varphi^{r-i} \phi^{i}}:=\mathcal{L} \otimes_{S}\left(S^{r}, \varphi^{r-i} \phi^{i}\right)
$$

where $\left(S^{r}, \varphi^{r-i} \phi^{i}\right)$ is $S^{r}$ viewed as an $S$-algebra via $\varphi^{r-i} \phi^{i}$. Furthermore, if $w \in W(r), w=\sum a_{i} \phi^{i}$, we define the $S^{r}$-module

$$
M_{X}^{r}(w):=\left(\mathcal{L}^{\varphi^{r}}\right)^{\otimes a_{0}} \otimes\left(\mathcal{L}^{\varphi^{r-1} \phi}\right)^{\otimes a_{1}} \otimes \cdots \otimes\left(\mathcal{L}^{\phi^{r}}\right)^{\otimes a_{r}} .
$$

We may call $M_{X}^{r}(w)$ the space of $\delta$-modular forms of weight $w$ on $X$. Replacing, in the definition above, $S^{r}$ by $S_{\text {for }}^{r}$ we may define $S_{\text {for }}^{r}$-modules

$$
M_{X}^{r} \text { for }(w):=\left(\mathcal{L}_{\text {for }}^{\varphi^{r}}\right)^{\otimes a_{0}} \otimes\left(\mathcal{L}_{\text {for }}^{\varphi^{r-1} \phi}\right)^{\otimes a_{1}} \otimes \cdots \otimes\left(\mathcal{L}_{\text {for }}^{\phi^{r}}\right)^{\otimes a_{r}} .
$$

We may call $M_{X^{\text {for }}}^{r}(w)$ the space of $\delta$-modular forms of weight $w$ on $X^{\text {for }}$.

\subsection{Isogeny covariant series}

For $\gamma \in \mathbb{Z}_{p}$ set

$$
\Gamma(T)=\Gamma_{\gamma}(T)=(1+T)^{\gamma}-1 \in \mathbb{Z}_{p}[[T]]
$$




\section{A. Buium}

Let $F \in S_{\text {for }}^{r}=R[[T]]\left[T^{\prime}, \ldots, T^{(r)}\right]^{\wedge}$ be a series and $m \geqslant 0$ an integer. Let us say that $F$ is isogeny covariant of weight $m$ if $F$ satisfies the functional equation

$$
F\left(\Gamma(T), \delta(\Gamma(T)), \ldots, \delta^{r}(\Gamma(T))\right)=\gamma^{m} \cdot F\left(T, T^{\prime}, \ldots, T^{(n)}\right)
$$

for all $\gamma \in \mathbb{Z}_{p}$. Recall from [Bui03, Theorem 3.11] that if $F$ satisfies Equation (1.3) for some $\gamma \in \mathbb{Z}_{p}$ which is not a root of unity then $F$ satisfies Equation (1.3) for all $\gamma \in \mathbb{Z}_{p}$. Also, by [Bui03, Theorem 3.11], we have that $F$ is isogeny covariant of weight $m$ if and only if there exists a homogeneous polynomial $\Phi \in K\left[x_{1}, \ldots, x_{r}\right]$ of degree $m$, such that

$$
F=\Phi\left(\Psi, \Psi^{\phi}, \ldots, \Psi^{\phi^{r-1}}\right),
$$

where $\Psi$ is the series

$$
\Psi:=\frac{1}{p} \log \frac{1+T^{p}+p T^{\prime}}{(1+T)^{p}} \in R[[T]]\left[T^{\prime}\right]^{\wedge} .
$$

So the isogeny covariant series of weight $m$ in $R[[T]]\left[T^{\prime}, \ldots, T^{(r)}\right]^{\wedge}$ form an $R$-module of rank $(m+r-1) ! /[m !(r-1) !]$.

\subsection{Formally isogeny covariant differential modular forms}

By 'functoriality', there is a naturally induced injective 'Serre-Tate' expansion map (at $P_{0}$ ):

$$
E: M_{X}^{r}(w) \rightarrow M_{X^{\text {for }}}^{r}(w) \simeq S_{\text {for }}^{r}=R[[T]]\left[T^{\prime}, \ldots, T^{(r)}\right]^{\wedge}
$$

(cf. the beginning of $\S 7$ for details). A differential modular form $g \in M_{X}^{r}(w)$ with $\operatorname{deg}(w)$ even will be called formally isogeny covariant (at $P_{0}$ ) if $E(g)$ is an isogeny covariant series of weight $m:=$ $-\operatorname{deg}(w) / 2$. (Our terminology is explained by the fact, to be exploited below, that if $g \in M_{X}^{r}(w)$ 'comes from' an isogeny covariant $\delta$-modular form of weight $w$, in the sense of [Bui03, $\S 1$ ], then $g$ is formally isogeny covariant; cf. [Bui03, Theorem 3.12].) Let us denote by $I_{X}^{r}(w)$ the $R$-submodule of all forms in $M_{X}^{r}(w)$ which are formally isogeny covariant (at $P_{0}$ ). The first main result of the present paper (Theorem 1.1) states that, if all $k$-points of $X$ are ordinary, then $I_{X}^{r}(w)$ has the maximum possible rank $(m+r-1) ! /[m !(r-1) !]$. Our second main result (Theorem 1.2) shows that the above fails in the presence of supersingular points. This phenomenon was first discovered by Barcau [Bar03] in the special case of differential modular forms on 'modular curves of level 1'; as already mentioned, his proofs depended on 'computations' in the coordinates $E_{4}$ and $E_{6}$. Our proofs will follow Barcau's strategy in [Bar03] but will require a coordinate free approach.

Theorem 1.1. Assume all $k$-points of $X$ are ordinary. Let $w \in W$ be such that

$$
\operatorname{deg}(w)=-2 m \in 2 \mathbb{Z}, \quad m \geqslant 0, \quad \operatorname{ord}(w) \leqslant r,
$$

and let $\Phi \in R\left[x_{1}, \ldots, x_{r}\right]$ be a homogeneous polynomial of degree $m$. Then there exists a $\delta$-modular form $g \in M_{X}^{r}(w)$ such that

$$
E(g)=\Phi\left(\Psi, \Psi^{\phi}, \ldots, \Psi^{\phi^{r-1}}\right) .
$$

In particular

$$
\operatorname{rank} I_{X}^{r}(w)=\frac{(m+r-1) !}{m !(r-1) !} .
$$

In the statement below for $v=\sum_{i=0}^{r-1} a_{i} \phi^{i} \in W_{+}$we write

$$
x^{v}=x_{1}^{a_{0}} \cdots x_{r}^{a_{r-1}} .
$$

Also we denote by ||$: K \rightarrow \mathbb{R}$ the $p$-adic absolute value such that, say, $|p|=p^{-1}$.

Theorem 1.2. Assume $X$ contains a supersingular $k$-point. Let $w \in W$ be such that

$$
\operatorname{deg}(w)=-2 m \in 2 \mathbb{Z}, \quad 0 \leqslant m<p, \quad w(p)<-2 m p, \quad \operatorname{ord}(w) \leqslant r .
$$




\section{Differential MODUlar FORMS ON SHIMURA CURVES, II}

Let $0 \neq g \in M_{X}^{r}(w)$ be a $\delta$-modular form such that

$$
E(g)=\Phi\left(\Psi, \Psi^{\phi}, \ldots, \Psi^{\phi^{r-1}}\right),
$$

where

$$
\Phi=\sum \lambda_{v} x^{v} \in K\left[x_{1}, \ldots, x_{r}\right]
$$

is a homogeneous polynomial of degree $m$. Then there exists $v$ such that

$$
\left|\lambda_{v}\right|>\left|\lambda_{m}\right|
$$

In particular $\Phi \notin K \cdot x_{1}^{m}$, hence

$$
\operatorname{rank} I_{X}^{r}(w)<\frac{(m+r-1) !}{m !(r-1) !}
$$

Theorem 1.2 shows that, in the presence of supersingular points, there are $p$-adic restrictions for the coefficients of the polynomials $\Phi$ that represent formally isogeny covariant forms. As we will see in this paper, for any fixed $D$, there exists $p_{0}$ such that for all primes $p \geqslant p_{0}$ there exists a Shimura curve $X$ containing a $k$-point that corresponds to an ordinary Jacobian and a supersingular $k$-point. Note that the condition $w(p)<-2 m p$ is essential; as we shall recall from [Bui03], Theorem 1.2 above fails for $w=-1-\phi$.

Theorem 1.2 will imply, for instance, the following corollary.

Corollary 1.3. Assume $X$ contains a supersingular $k$-point. Then the following hold:

1) The $R$-modules $I_{X}^{1}(-1-\phi), I_{X}^{2}\left(-1-\phi^{2}\right)$, and $I_{X}^{2}\left(-\phi-\phi^{2}\right)$ have rank one.

2) The R-modules $I_{X}^{r}(w)$ vanish for all $w$ with $\operatorname{deg}(w)=0$ and either $w(p)<0$ or $0<w(p) \leqslant$ $p^{\operatorname{ord}(\bar{w})}-p^{\operatorname{ord}(\bar{w})-1}$.

As explained in [Bui03] the weights $-1-\phi$ and $-1-\phi^{2}$ in assertion 4 of Theorem 1.4 are the two 'basic weights' in this theory.

\subsection{Application to isogeny covariant differential modular forms}

Fix a quaternion algebra $D$ and a sufficiently big prime $p$. For any $w \in W$ of even degree we defined in [Bui03] the $\mathbb{Z}_{p}$-module $I_{D}(w)$ of isogeny covariant $\delta$-modular forms on $D$ of weight $w$ (cf. [Bui03, $\S 1.8]$. We have the following theorem.

\section{THEOREM 1.4.}

1) The $\mathbb{Z}_{p}$-modules $I_{D}(w)$ are finitely generated.

2) $I_{D}(w)=0$ for $\operatorname{deg}(w)>0$.

3) $I_{D}(0)=\mathbb{Z}_{p}$.

4) The $R$-modules $I_{D}(-1-\phi), I_{D}\left(-1-\phi^{2}\right)$, and $I_{D}\left(-\phi-\phi^{2}\right)$ have rank one.

5) The $R$-modules $I_{D}(w)$ vanish for all $w$ with $\operatorname{deg}(w)=0$, and either $w(p)<0$ or $0<w(p) \leqslant$ $p^{\operatorname{ord}(\bar{w})}-p^{\operatorname{ord}(\bar{w})-1}$.

Assertions 1-3 in Theorem 1.4 (and also assertion 4 for $I_{D}(-1-\phi)$ ) were proved in [Bui03, Theorem 1.2]. The rest of the assertions will be proved in the present paper. Note that assertion 4 in Theorem 1.4 proves, in particular, the last assertion in Theorem 1.2 [Bui03], which was stated there without proof. 


\section{A. BuIUM}

\section{Normalized basis of $H^{1}$}

Assume first $X$ is a Shimura curve. Let us consider the $\mathcal{O}_{D}$-equivariant exact sequence of $S$-modules

$$
0 \rightarrow H^{10} \rightarrow H^{1} \rightarrow H^{01} \rightarrow 0
$$

where

$$
H^{10}:=H^{0}\left(A, \Omega_{A / S}^{1}\right), \quad H^{1}:=H_{D R}^{1}(A / S), \quad H^{01}:=H^{1}(A, \mathcal{O}),
$$

and let us choose an $\mathcal{O}_{D^{-}}$-equivariant right inverse

$$
\tau: H^{01} \rightarrow H^{1}
$$

to the projection $\pi: H^{1} \rightarrow H^{01}$; this will induce an $\mathcal{O}_{D^{-}}$equivariant splitting of $(2.1)$. Such an $\mathcal{O}_{D}$-equivariant right inverse always exists; indeed, if $\tau^{\prime}$ is any right inverse of $H^{1} \rightarrow H^{01}$ then

$$
\tau:=e_{11} \tau^{\prime} e_{11}+e_{12} \tau^{\prime} e_{21}
$$

is an $\mathcal{O}_{D}$-equivariant right inverse. In the above formula,

$$
e_{11}, e_{12}, e_{21}, e_{22} \in \operatorname{End}_{S}\left(H^{1}\right)
$$

are the images of the natural idempotents via the map

$$
\operatorname{Mat}_{2}(S)^{\text {op }} \simeq \mathcal{O}_{D}^{\text {op }} \otimes_{\mathbb{Z}} S \rightarrow \operatorname{End}_{S}\left(H^{1}\right),
$$

where the isomorphism above is induced by the isomorphism (1.2).

At various points in this paper we are going to make constructions depending 'functorially' (in a sense to be made precise later) on $(X, \tau)$. Some of the constructions will actually not depend on $\tau$ and we are going to point out the situations when this happens.

Define

$$
\mathcal{L}:=e_{11} H^{10}, \quad \mathcal{H}:=e_{11} H^{1}, \quad \mathcal{Q}:=e_{11} H^{01} .
$$

(Note that our notations are at variance with those in [DT94].) The restriction of the map (2.2) induces a right inverse $\tau: \mathcal{Q} \rightarrow \mathcal{H}$ to the projection $\mathcal{H} \rightarrow \mathcal{Q}$; we set $\mathcal{L}^{\prime}:=\tau(\mathcal{Q})$.

Replacing $S$ by $S_{\text {for }}$ in the constructions above we have $S_{\text {for-modules }}$

$$
H_{\text {for }}^{1}, \mathcal{L}_{\text {for }}, \mathcal{H}_{\text {for }}, \mathcal{Q}_{\text {for }}, \mathcal{L}_{\text {for }}^{\prime}
$$

If $X$ is a modular curve then, since 6 is invertible in $R$, the exact sequence (2.1) has a splitting $\tau: H^{01} \rightarrow H^{1}$; cf. [Kat73b, p. 163]. We set, in this case,

$$
\mathcal{L}:=H^{10}, \quad \mathcal{H}:=H^{1}, \quad \mathcal{Q}:=H^{01}, \quad \mathcal{L}^{\prime}:=\tau(\mathcal{Q}) .
$$

We define corresponding $S_{\text {for-modules }} H_{\text {for }}^{1}, \mathcal{L}_{\text {for }}, \ldots$

Going back to the case $X$ is a Shimura curve, let $\omega^{1}$ be a local basis of $\mathcal{L}$ and $\omega=\left(\omega^{1}, \omega^{2}\right)^{\mathrm{t}}$ the corresponding false 1-form in the sense of [Bui03, $\S 1.4$ ]; in particular $\omega^{2}=e_{12} \omega^{1}$. (Here the t superscript denotes the transpose of a row vector.) The commutative diagrams

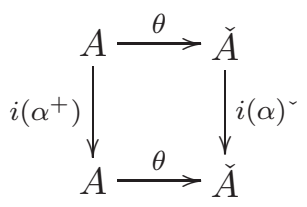




\section{Differential modular forms on Shimura CURVEs, II}

for $\alpha \in \mathcal{O}_{D}$, induce commutative diagrams

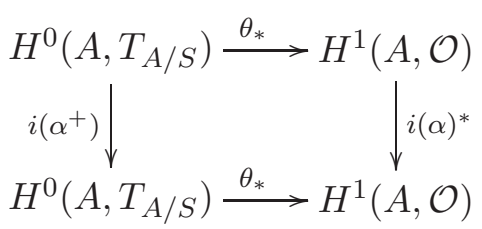

at the level of Lie algebras; here + is the involution considered in [Bui03, $\S 1.2]$. Let $\xi=\left(\xi^{1}, \xi^{2}\right)^{\mathrm{t}}$ be the unique local basis of $H^{1}(A, \mathcal{O})$ such that

$$
\left\langle\theta_{*}^{-1} \xi, \omega^{\mathrm{t}}\right\rangle=I
$$

where $I$ is the identity matrix; we call $\xi$ the $\theta$-dual of $\omega$. We claim that the following formula holds:

$$
i(\alpha)^{*} \xi=j\left(\alpha^{+}\right)^{\mathrm{t}} \xi, \quad \alpha \in \mathcal{O}_{D} .
$$

Indeed, if $i(\alpha)^{*} \xi=k(\alpha) \xi$ then

$$
\begin{aligned}
j\left(\alpha^{+}\right)^{\mathrm{t}} & =\left\langle\theta_{*}^{-1} \xi, \omega^{\mathrm{t}} j\left(\alpha^{+}\right)^{\mathrm{t}}\right\rangle=\left\langle\theta_{*}^{-1} \xi, i\left(\alpha^{+}\right)^{*} \omega^{\mathrm{t}}\right\rangle \\
& =\left\langle i\left(\alpha^{+}\right) \theta_{*}^{-1} \xi, \omega^{\mathrm{t}}\right\rangle=\left\langle\theta_{*}^{-1} i(\alpha)^{*} \xi, \omega^{\mathrm{t}}\right\rangle=k(\alpha),
\end{aligned}
$$

which proves (2.7). Note that by our normalizations in [Bui03, $\S 1.2]$,

$$
j\left(\alpha^{+}\right)^{\mathrm{t}}=\left(\begin{array}{cc}
d & 0 \\
0 & 1
\end{array}\right) j(\alpha)\left(\begin{array}{cc}
d^{-1} & 0 \\
0 & 1
\end{array}\right),
$$

where $d$ is the discriminant of $D$. In particular (2.7) shows that

$$
e_{11} \xi^{1}=\xi^{1}, \quad e_{22} \xi^{2}=\xi^{2}, \quad e_{12} \xi^{1}=d \xi^{2},
$$

so $\xi^{1}$ is a local basis of $\mathcal{Q}$. Define

$$
\eta^{1}:=\tau\left(\xi^{1}\right), \quad \eta^{2}:=\tau\left(\xi^{2}\right)
$$

by $\mathcal{O}_{D}$-equivariance of $\tau$ we have

$$
e_{11} \eta^{1}=\eta^{1}, \quad e_{22} \eta^{2}=\eta^{2}, \quad e_{12} \eta^{1}=d \eta^{2}
$$

in particular $\eta^{1}$ is a local basis of $\mathcal{L}^{\prime}$. Note that

$$
\omega^{1}, \eta^{1}, \omega^{2}, \eta^{2}
$$

is a local basis of $H^{1}$. A local basis of this form will be called normalized; it is uniquely determined by its first vector $\omega^{1}$. Note that

$$
\left\langle\eta, \omega^{\mathrm{t}}\right\rangle_{\theta}=I
$$

where $\langle,\rangle_{\theta}$ is the bilinear, antisymmetric pairing on $H^{1}$ induced by $\theta$ (cf. [FC90, p. 81]). Recall that, $(A, \theta)$ is isomorphic to the Jacobian of a curve then $\langle,\rangle_{\theta}$ identifies with the cup product on the curve.

If $X$ is a modular curve, $\omega=\omega^{1}$ is a local basis of $\mathcal{L}$, and $\xi=\xi^{1}$ satisfies Equation (2.6) then we set $\eta^{1}:=\tau(\xi)$ and we refer to

$$
\omega^{1}, \eta^{1}
$$

as a normalized basis for $H^{1}$.

One defines normalized bases of $H_{\text {for }}^{1}$ similarly; we use the same notations as above with for as a subscript: $\omega_{\text {for }}^{1}, \ldots$. When no confusion is likely to arise we will drop, however, the for subscript. 


\section{A. BuIUM}

\section{The operators $\partial$ and $\partial_{r}$}

Let $X$ be either a Shimura curve or a modular curve.

Let $\hat{\Omega}$ denote the $p$-adic completion of $\Omega_{S / R}^{1}$ and let $\Omega^{\text {for }}=R[[T]] d T$. Recall that we have at our disposal the Gauss-Manin connection

$$
\nabla: H^{1} \rightarrow H^{1} \otimes \hat{\Omega}
$$

By the functoriality of the Gauss-Manin connection, $\nabla$ commutes with $e_{i j}$ so (3.1) induces a connection

$$
\nabla: \mathcal{H} \rightarrow \mathcal{H} \otimes \hat{\Omega} .
$$

If $X$ is a Shimura curve then, with respect to a basis as in (2.9), we may write (3.1) as

$$
\left(\begin{array}{c}
\nabla \omega^{1} \\
\nabla \eta^{1} \\
\nabla \omega^{2} \\
\nabla \eta^{2}
\end{array}\right)=\left(\begin{array}{cccc}
\sigma_{11} & \sigma_{12} & 0 & 0 \\
\sigma_{21} & \sigma_{22} & 0 & 0 \\
0 & 0 & \sigma_{11} & \sigma_{12} d \\
0 & 0 & \sigma_{21} d^{-1} & \sigma_{22}
\end{array}\right)\left(\begin{array}{c}
\omega^{1} \\
\eta^{1} \\
\omega^{2} \\
\eta^{2}
\end{array}\right)
$$

where $\sigma_{i j} \in \hat{\Omega}$. Similarly, if $X$ is a modular curve, we have

$$
\left(\begin{array}{c}
\nabla \omega^{1} \\
\nabla \eta^{1}
\end{array}\right)=\left(\begin{array}{ll}
\sigma_{11} & \sigma_{12} \\
\sigma_{21} & \sigma_{22}
\end{array}\right)\left(\begin{array}{l}
\omega^{1} \\
\eta^{1}
\end{array}\right) .
$$

In particular the 'Kodaira-Spencer matrix'

$$
\left\langle\nabla \omega, \omega^{\mathrm{t}}\right\rangle_{\theta}
$$

equals

$$
\sigma_{12} \cdot\left(\begin{array}{ll}
1 & 0 \\
0 & d
\end{array}\right)
$$

in the Shimura curve case and equals $\sigma_{12}$ in the modular curve case. Since $A$ is formally universal at all $k$-points, the reduction mod $p$ of the matrix (3.5) is nowhere vanishing. It follows that $\sigma_{12}$ is a nowhere vanishing 1 -form. Note that $\sigma_{12}$ is independent of the choice of $\tau$.

We claim now that the bilinear maps

$$
\begin{gathered}
\mathcal{L} \times \mathcal{L} \rightarrow \hat{\Omega}, \quad \mathcal{L}^{\prime} \times \mathcal{L} \rightarrow S \\
(x, y) \mapsto\langle\nabla x, y\rangle_{\theta}
\end{gathered}
$$

induce isomorphisms

$$
\mathcal{L}^{\otimes 2} \simeq \hat{\Omega}, \quad \mathcal{L}^{\otimes(-1)} \simeq \mathcal{L}^{\prime}
$$

(The isomorphisms (3.7) are well known; cf. [DT94, Lemma 7, p. 454], for Shimura curves and [Kat73b] for modular curves; we will reprove them here in order to review the explicit bilinear maps (3.6) that realize them.) Indeed, in the Shimura curve case, it is enough to check (3.7) in a local normalized basis (2.9). In such a basis, however, the bilinear maps (3.6) send

$$
\left(\omega^{1}, \omega^{1}\right) \mapsto \sigma_{12}, \quad\left(\eta^{1}, \omega^{1}\right) \mapsto 1,
$$

and we are done. Of course, the isomorphisms (3.7) are independent of $\tau$. A similar argument works for modular curves.

For the rest of this section we let $X$ be either a Shimura curve or a modular curve.

The connection (3.2) induces a connection

$$
\nabla: \operatorname{Symm}(\mathcal{H}) \rightarrow \operatorname{Symm}(\mathcal{H}) \otimes \hat{\Omega}
$$




\section{Differential MODUlar FORMS ON SHIMURA CURVES, II}

on the symmetric algebra of $\mathcal{H}$. Using the natural inclusion $\mathcal{L} \rightarrow \mathcal{H}$, its right inverse given by the second projection $\pi: \mathcal{H}=\mathcal{L} \oplus \mathcal{L}^{\prime} \rightarrow \mathcal{L}$, and the isomorphism (3.7), we get an $R$-derivation $\partial=\partial^{\tau}$, depending on the splitting map $\tau$ in $(2.2)$ :

$$
\begin{aligned}
\partial: \bigoplus_{n \geqslant 0} \mathcal{L}^{\otimes n}= & \operatorname{Symm}(\mathcal{L}) \rightarrow \operatorname{Symm}(\mathcal{H}) \stackrel{\nabla}{\rightarrow} \operatorname{Symm}(\mathcal{H}) \otimes \hat{\Omega}_{S / R} \simeq \operatorname{Symm}(\mathcal{H}) \otimes \mathcal{L}^{\otimes 2} \\
& \rightarrow \operatorname{Symm}(\mathcal{H}) \otimes \operatorname{Symm}(\mathcal{H}) \stackrel{\operatorname{mult}}{\rightarrow} \operatorname{Symm}(\mathcal{H}) \stackrel{\pi}{\rightarrow} \operatorname{Symm}(\mathcal{L})=\bigoplus_{n \geqslant 0} \mathcal{L}^{\otimes n} .
\end{aligned}
$$

If we choose a normalized local basis (2.9) or (2.10) (according as $X$ is a Shimura curve or a modular curve) then $\bigoplus_{n \geqslant 0} \mathcal{L}^{\otimes n}$ is locally isomorphic to a ring of polynomials $S[x]$, where the variable $x$ corresponds to $\omega^{1}$, and the derivation $\partial$ satisfies

$$
\begin{gathered}
\partial(x)=\frac{\sigma_{11}}{\sigma_{12}} x^{3} \\
\partial(s)=\frac{d s}{\sigma_{12}} x^{2}, \quad s \in S .
\end{gathered}
$$

(The above formulae make sense because $\sigma_{12}$ is an invertible 1-form. Note also that the value of $\partial(s)$ does not depend on the choice of $\tau$.) In particular $\partial\left(\mathcal{L}^{\otimes n}\right) \subset \mathcal{L}^{\otimes(n+2)}$. (If $X$ is a modular curve then the $R$-derivation $\partial$ is, of course, the Serre operator for modular curves; cf. [Kat73b, Appendix].) We claim that $\partial$ above uniquely extends to an $R$-derivation:

$$
\partial: \bigoplus_{n \in \mathbb{Z}} \mathcal{L}^{\otimes n} \rightarrow \bigoplus_{n \in \mathbb{Z}} \mathcal{L}^{\otimes n}
$$

Indeed, by the uniqueness part of the claim, it is enough to check the claim locally on $S$; but, locally on $S, \bigoplus_{n \in \mathbb{Z}} \mathcal{L}^{\otimes n}$ is a ring of fractions of $\bigoplus_{n \geqslant 0} \mathcal{L}^{\otimes n}$ (because $R\left[x, x^{-1}\right]$ is a ring of fractions of $R[x]$ ) and the claim is clearly true in this case.

All that was said so far in this section can be done for the Gauss-Manin connection

$$
\nabla^{\text {for }}: \mathcal{H}_{\text {for }} \rightarrow \mathcal{H}_{\text {for }} \otimes \Omega^{\text {for }}
$$

in place of $\nabla$; one just has to put subscripts (or, if one prefers, superscripts) 'for' to all objects involved.

Recall that we defined in $\S 1$ the $S^{r}$-module $M_{X}^{r}(w)$ of $\delta$-modular forms of weight $w$ on $X$ and the $S_{\text {for }}^{r}$-module $M_{X \text { for }}^{r}(w)$ of $\delta$-modular forms of weight $w$ on $X^{\text {for }}$. We will also need to consider the space

$$
\bar{M}_{X}^{r}(w):=M_{X}^{r}(w) \otimes_{R} k
$$

which we call the space of $\delta$-modular forms of weight $w$ on $X \bmod p$. Let $M(w)=M_{D}(w)$ be the space of $\delta$-modular forms of weight $w$ on $D$ (in the sense of [Bui03, $\S 1.8]$ ) or $M(w)=M_{1}(w)$ be the space of $\delta$-modular forms of weight $w$ and genus 1 (in the sense of [Bui03, $\S 1.7$ ]) according as $X$ is a Shimura curve or a modular curve. Then there are natural $R$-module maps

$$
M(w) \otimes_{\mathbb{Z}_{p}} R \rightarrow M_{X}^{r}(w)
$$

defined as follows. Let $f \in M(w)$ and let $x=\omega^{1}$ be a local basis of $\mathcal{L}$; then, in the Shimura curve case, consider the false 1 -form $\omega:=\left(\omega^{1}, \omega^{2}\right)^{\mathrm{t}}$, where $\omega^{2}:=e_{12} \omega^{1}$, and consider the element of $M_{X}^{r}(w)$ defined locally by

$$
f(A, i, \theta, \omega) \cdot x^{\otimes w}
$$

where, if $w=\sum a_{i} \phi^{i}$, then

$$
x^{\otimes w}=x^{w}:=\prod\left(x^{\varphi^{r-i} \phi^{i}}\right)^{a_{i}} .
$$




\section{A. BuIUM}

The 'local section' (3.13) does not depend on the choice of $\omega^{1}$ and defines a 'global' element in $M_{X}^{r}(w)$ which we take to be the image of $f$ in $M_{X}^{r}(w)$. A similar definition can be given in the modular curve case. Note also that we have natural ring homomorphisms

$$
\varphi, \phi: \bigoplus_{w \in W(r-1)} M_{X}^{r-1}(w) \rightarrow \bigoplus_{w \in W(r)} M_{X}^{r}(w)
$$

induced by the homomorphisms

$$
\varphi: M_{X}^{r-1}(w) \rightarrow M_{X}^{r}(w), \quad \phi: M_{X}^{r-1}(w) \rightarrow M_{X}^{r}(\phi w) .
$$

As a rule we shall view $\varphi$ as an inclusion. Let us define a sequence of rings $\left(M_{X}^{r}\right)$ by

$$
M_{X}^{r}=\mathcal{O}\left(J^{r}\left(\operatorname{Spf}\left(\left(\bigoplus_{m \in \mathbb{Z}} \mathcal{L}^{\otimes m}\right)\right)\right)\right),
$$

where $J^{r}$ denotes, as usual, the $p$-jet space of order $r$ of a smooth formal scheme; cf. $\S 1.4$. Similarly we define a prolongation sequence $\left(M_{X^{\text {for }}}^{r}\right)$ as follows. We choose a basis $x_{\text {for }}$ of $\mathcal{L}_{\text {for }}$; when it is clear that we are dealing with the formal case we will usually write $x$ in place of $x_{\text {for }}$. (However, note that, typically, the basis $x_{\text {for }}$ will not come from a local basis of $\mathcal{L}$ !) We let

$$
\bigoplus_{m \in \mathbb{Z}} \mathcal{L}_{\mathrm{for}}^{\otimes m} \simeq R[[T]]\left[x, x^{-1}\right]
$$

be the corresponding isomorphism and we set

$$
M_{X^{\text {for }}}^{r}:=R[[T]]\left[x, x^{-1}, x^{\prime}, \ldots, x^{(r)}, T^{\prime}, \ldots, T^{(r)}\right]^{\wedge} .
$$

The $S_{\text {for }}^{r}$-algebras $M_{X \text { for }}^{r}$ can be called the spaces of $\delta$-modular functions on $X^{\text {for }}$. We also let

$$
\bar{M}_{X^{\text {for }}}^{r}:=M_{X}^{r} \text { for } \otimes_{R} k
$$

and call this the space of $\delta$-modular functions on $X^{\text {for }} \bmod p$.

Proposition 3.1. Fix $r \geqslant 1$. Then the following hold.

i) There are natural inclusions

$$
\bigoplus_{w \in W(r)} M_{X}^{r}(w) \subset M_{X}^{r}
$$

inducing inclusions

$$
\bar{M}_{X}^{r}(w) \subset \bar{M}_{X}^{r}
$$

for each $w$.

ii) There is a unique derivation

$$
\partial_{r}: M_{X}^{r} \rightarrow M_{X}^{r},
$$

depending on $\tau$, with the following properties:

1) $\partial_{r}$ vanishes on $M_{X}^{r-1}$,

2) $\partial_{r} \circ \delta^{r}=\phi^{r} \circ \partial$ on $\bigoplus_{m \in \mathbb{Z}} \mathcal{L}^{\otimes m}$, and

3) $\partial_{r}\left(M_{X}^{r}(w)\right) \subset M_{X}^{r}\left(w+2 \phi^{r}\right)$.

iii) The $\bar{M}_{X}^{r-1}$-derivation

$$
\bar{\partial}_{r}: \bar{M}_{X}^{r} \rightarrow \bar{M}_{X}^{r}
$$

obtained by reducing $\partial_{r}$ modulo $p$ has the property that its restriction to any of the spaces $\bar{M}_{X}^{r}(w)$ does not depend on $\tau$.

iv) If an element $f \in M_{X}^{r}(w)$ is invertible in $M_{X}^{r}$ then its inverse lies in $M_{X}^{r}(-w)$. 


\section{Differential MOdUlar Forms on Shimura CURVES, II}

v) If $X_{1} \subset X$ is an affine open subscheme then

$$
M_{X}^{r} \cap M_{X_{1}}^{r}(w)=M_{X}^{r}(w) .
$$

Before we give the proof, some remarks are in order.

Remark 3.2. As will be clear from the proof:

1) $M_{X}^{r}$ is not the $p$-adic closure of $\bigoplus_{w \in W(r)} M_{X}^{r}(w)$.

2) The induced map $\bigoplus_{w \in W(r)} \bar{M}_{X}^{r}(w) \rightarrow \bar{M}_{X}^{r}$ is not injective.

Let us also note that:

3) Conditions 1 and 2 in Proposition 3.1 formally imply that

$$
\partial_{r} \circ \phi^{r}=p^{r} \phi^{r} \circ \partial \text { on } \bigoplus_{m \in \mathbb{Z}} \mathcal{L}^{\otimes m} .
$$

4) A statement identical to that of Proposition 3.1 holds for $X, \mathcal{L}$ replaced by $X^{\text {for }}, \mathcal{L}_{\text {for }}$.

We need the following general construction in [BZ04].

Lemma 3.3 [BZ04]. Let $\left(B^{r}\right)$ be a prolongation sequence such that either

i) $B$ is the $p$-adic completion of a smooth $R$-algebra and $J^{r}(\operatorname{Spf} B)=\operatorname{Spf} B^{r}$ are the $p$-jet spaces of $\operatorname{Spf} B$ or

ii) $B^{r}=R[[T]]\left[x, x^{-1}, x^{\prime}, \ldots, x^{(r)}, T^{\prime}, \ldots, T^{(r)}\right]^{\wedge}, B:=B^{0}$.

Let $\partial: B \rightarrow B$ be an $R$-derivation. Then, for each $r \geqslant 1$, there exists a unique derivation

$$
\partial_{r}: B^{r} \rightarrow B^{r}
$$

with the following properties:

1) $\partial_{r}$ vanishes on $B^{r-1}$,

2) $\partial_{r} \circ \delta^{r}=\phi^{r} \circ \partial$ on $B$.

Proof of Proposition 3.1. The proposition is, clearly, a local statement on $X$. So we may, and will, assume that $\mathcal{L}$ is a free $S$-module and, hence, upon choosing a basis $x$ of $\mathcal{L}$, we have induced identifications

$$
\begin{gathered}
\bigoplus_{m \in \mathbb{Z}} \mathcal{L}^{\otimes m}=S\left[x, x^{-1}\right], \\
\bigoplus_{w \in W(r)} M_{X}^{r}(w)=B_{r}:=S^{r}\left[x, x^{-1}, \phi x, \phi x^{-1}, \ldots, \phi^{r} x, \phi^{r} x^{-1}\right],
\end{gathered}
$$

where $\phi^{i} x$ are variables and $\phi$ sends $\phi^{i} x$ into $\phi^{i+1} x$, for $i \leqslant r-1$. Set

$$
B:=S\left[x, x^{-1}\right]^{\wedge} \text {. }
$$

Then, by continuity, the derivation

$$
\partial: S\left[x, x^{-1}\right] \rightarrow S\left[x, x^{-1}\right]
$$

induces an $R$-derivation $\partial: B \rightarrow B$. Let $B^{r}:=M_{X}^{r}$, i.e. Spf $B^{r}:=J^{r}(\operatorname{Spf} B)$. By the general properties of $p$-jet spaces in [Bui00, pp. 104-105],

$$
B^{r}=S^{r}\left[x, x^{-1}, x^{\prime}, \ldots, x^{(r)}\right]^{\wedge},
$$

where $x^{\prime}, \ldots, x^{(r)}$ are new variables with $\delta x^{(i)}=x^{(i+1)}$. The ring $B_{r}$ in (3.17) is embedded into the ring $B^{r}$ of (3.18) via

$$
\phi x \mapsto x^{p}+p x^{\prime}, \quad \phi^{2} x \mapsto\left(x^{p}+p x^{\prime}\right)^{p}+p\left(\left(x^{\prime}\right)^{p}+p x^{\prime \prime}\right), \quad \ldots
$$




\section{A. Buium}

Note that if $\bar{S}^{r}:=S^{r} / p S^{r}$ then the image of $\bar{M}_{X}^{r}(w)=\bar{S}^{r} \cdot \bar{x}^{\otimes w}$ in $\bar{M}_{X}^{r}$ equals $\bar{S}^{r} \cdot \bar{x}^{w(p)}$ so the map $\bar{M}_{X}^{r}(w) \rightarrow \bar{M}_{X}^{r}$ is injective. This proves assertion i in Proposition 3.1.

By Lemma 3.3 there is a derivation

$$
\partial_{r}: B^{r} \rightarrow B^{r}
$$

satisfying the properties listed there. We claim that $\partial_{r}$ sends the ring $B_{r}$ into itself. To check the claim, note first that, by property 1 in Lemma 3.3, we have $\partial\left(S^{r-1}\right)=0$ and

$$
\partial_{r}(x)=\cdots=\partial_{r}\left(\phi^{r-1} x\right)=0,
$$

which implies, of course, that

$$
\partial_{r}\left(x^{-1}\right)=\cdots=\partial_{r}\left(\phi^{r-1} x^{-1}\right)=0 .
$$

Also, for $s \in S$, Equation (3.11) implies that

$$
\partial_{r}\left(\delta^{r} s\right)=\phi^{r}(\partial(s))=\phi^{r}\left(\frac{d s}{\sigma_{12}} x^{2}\right)=\phi^{r}\left(\frac{d s}{\sigma_{12}}\right)\left(\phi^{r} x\right)^{2} \in S^{r} \cdot\left(\phi^{r} x\right)^{2} .
$$

Note that $\partial_{r}\left(\delta^{r} s\right)$ is independent of the choice of $\tau$. Since any element of $S^{r}$ is a $p$-adic limit of $S^{r-1}$-linear combinations of products of elements of the form $\delta^{r} s$, with $s \in S$, it follows easily that

$$
\partial_{r} s_{r} \in S^{r} \cdot\left(\phi^{r} x\right)^{2}
$$

for all $s_{r} \in S^{r}$ and that the value of $\partial_{r} s_{r}$ does not depend on the choice of $\tau$. Finally, Equation (3.10) implies that, for any $m \in \mathbb{Z}$,

$$
\begin{aligned}
\partial_{r}\left(\left(\phi^{r} x\right)^{m}\right) & =\partial_{r}\left(\phi^{r}\left(x^{m}\right)\right)=p^{r} \phi^{r}\left(\partial\left(x^{m}\right)\right)=p^{r} \phi^{r}\left(m \frac{\sigma_{11}}{\sigma_{12}} x^{m+2}\right) \\
& =m p^{r} \phi^{r}\left(\frac{\sigma_{11}}{\sigma_{12}}\right)\left(\phi^{r} x\right)^{m+2} .
\end{aligned}
$$

Note that the value of $\partial_{r}\left(\left(\phi^{r} x\right)^{m}\right)$ really depends on the choice of $\tau$; but its reduction $\bmod p$ vanishes, so, in particular, it does not depend on $\tau$. This ends the proof of our claim and hence the construction of $\partial_{r}$. Assertions 1 and 2 in part ii of Proposition 3.1 have already been checked. Assertion 3 in part ii as well as part iii of Proposition 3.1 follow immediately from (3.19) and (3.20).

To prove assertion iv of Proposition 3.1, we, again, note that this is a local matter so we are reduced to showing that if an element $s \in S^{r}$ is such that $s \cdot x^{w}$ is invertible in the ring $S^{r}\left[x, x^{-1}, x^{\prime}, \ldots, x^{(r)}\right]^{\wedge}$ then $s$ is invertible in $S^{r}$ (and hence $s^{-1} \cdot x^{-w}$ is the inverse of $s \cdot x^{w}$ ). Write

$$
s \cdot x^{w} \cdot\left(\sum s_{i_{0} i_{1} \ldots i_{r}} x^{i_{0}}\left(x^{\prime}\right)^{i_{1}} \cdots\left(x^{(r)}\right)^{i_{r}}\right)=1,
$$

with all $s_{i_{0} i_{1} \ldots i_{r}}$ in $S^{r}$. Denoting by upper bar classes in $\bar{S}^{r}:=S^{r} / p S^{r}$ we obtain

$$
\bar{s} \cdot\left(\sum \bar{s}_{i_{0}, i_{1}, \ldots, i_{r}} x^{i_{0}+w(p)}\left(x^{\prime}\right)^{i_{1}} \cdots\left(x^{(r)}\right)^{i_{r}}\right)=1,
$$

and hence we get

$$
\bar{s} \cdot \bar{s}_{-w(p), 0, \ldots, 0}=1 \quad \text { in } \bar{S}^{r} .
$$

So $\bar{s}$ is invertible in $\bar{S}^{r}$; since $S^{r}$ is $p$-adically complete, it follows that $s$ itself is invertible in $S^{r}$.

Assertion v of Proposition 3.1 is again local so we need to show that if $S_{1}=\mathcal{O}\left(X_{1}\right)^{\wedge}$ and $s_{1} \in S_{1}^{r}$ is such that $s_{1} \cdot x^{w}$ belongs to $S^{r}\left[x, x^{-1}, x^{\prime}, \ldots, x^{(r)}\right]^{\wedge}$ then $s_{1} \in S^{r}$. Write $w=\sum a_{i} \phi^{i}$,

$$
\begin{gathered}
s_{1} \cdot x^{w}=s_{1} \cdot x^{a_{0}}\left(x^{p}+p x^{\prime}\right)^{a_{1}} \cdots\left(\left(x^{(r)}\right)^{p}+p x^{(r+1)}\right)^{a_{r}}, \\
s_{1} \cdot x^{w}=\sum s_{i_{0} i_{1} \ldots i_{r}} x^{i_{0}}\left(x^{\prime}\right)^{i_{1}} \cdots\left(x^{(r)}\right)^{i_{r}},
\end{gathered}
$$




\section{Differential modular forms on Shimura Curves, II}

with all $s_{i_{0} i_{1} \ldots i_{r}}$ in $S^{r}$. Picking out the coefficient of

$$
x^{a_{0}}\left(x^{\prime}\right)^{a_{1}} \cdots\left(x^{(r+1)}\right)^{a_{r}},
$$

we get that $p^{\operatorname{deg}(w)} \cdot s_{1}=s_{a_{0} a_{1} \ldots a_{r}} \in S^{r}$. Since $p^{n} S_{1}^{r} \cap S^{r}=p^{n} S^{r}$ (cf. Lemma 4.2 below) we get that $s_{1} \in S^{r}$.

Note that all our constructions above are functorial in $(X, \tau)$, in the following sense. Assume we have pairs $\left(X_{i}, \tau_{i}\right), i=1,2$, and an open immersion $X_{2} \rightarrow X_{1}$ of schemes such that the pull-back of $\tau_{1}$ is $\tau_{2}$. Then it is easy to check that there is a natural ring homomorphism $M_{X_{1}}^{r} \rightarrow M_{X_{2}}^{r}$ that is compatible with weights and with the actions of $\partial_{r}$ and $\phi$. Similarly if we have a pair $(X, \tau)$ and a $k$-point $P_{0}$ then there is a natural ring homomorphism $M_{X}^{r} \rightarrow M_{X^{\text {for }}}^{r}$ that is compatible with weights and with the actions of $\partial_{r}$ and $\phi$.

\section{The Hasse invariant}

Assume first that $X$ is a Shimura curve.

Let $(\bar{A}, \bar{i}, \bar{\theta}), \overline{\mathcal{L}}, \bar{\omega}$ and $\bar{\xi}$ be obtained, from $(A, i, \theta)$, a false 1 -form $\omega$, and its $\theta$-dual $\xi$ (cf. Equation (2.6)), by base change to $\bar{S}:=S / p S$. Note that the semilinear map

$$
F^{*}: H^{1}(\bar{A}, \mathcal{O}) \rightarrow H^{1}(\bar{A}, \mathcal{O})
$$

induced by the absolute $p$-power Frobenius on $\bar{A}$ commutes with all $\bar{S}$-linear endomorphisms

$$
i(\alpha)^{*}: H^{1}(\bar{A}, \mathcal{O}) \rightarrow H^{1}(\bar{A}, \mathcal{O}) .
$$

Consider the Hasse-Witt matrix $\bar{h} \in \operatorname{Mat}_{2}(\bar{S})$ of $\bar{A}$ with respect to the basis $\bar{\xi}$; hence, by definition,

$$
F^{*} \bar{\xi}=\bar{h} \bar{\xi}
$$

Let

$$
\mathbb{Z}_{p} \rightarrow \boldsymbol{F}_{p}, \quad x \mapsto c(x)
$$

be the canonical projection. Then, for all $\alpha \in \mathcal{O}_{D}$, we have

$$
\begin{aligned}
\bar{h} c\left(j\left(\alpha^{+}\right)^{\mathrm{t}}\right) \bar{\xi} & =\bar{h} i(\alpha)^{*} \bar{\xi}=i(\alpha)^{*} \bar{h} \bar{\xi}=i(\alpha)^{*} F^{*} \bar{\xi}=F^{*} i(\alpha)^{*} \bar{\xi} \\
& =F^{*}\left(c\left(j\left(\alpha^{+}\right)^{\mathrm{t}}\right) \bar{\xi}\right)=F\left(c\left(j\left(\alpha^{+}\right)^{\mathrm{t}}\right)\right) F^{*} \bar{\xi}=c\left(j\left(\alpha^{+}\right)^{\mathrm{t}}\right) \bar{h} \bar{\xi} .
\end{aligned}
$$

We conclude that

$$
\bar{h} c\left(j\left(\alpha^{+}\right)^{\mathrm{t}}\right)=c\left(j\left(\alpha^{+}\right)^{\mathrm{t}}\right) \bar{h}
$$

for all $\alpha$. This forces $\bar{h}$ to be a scalar matrix, $\bar{h} \in \bar{S}$. Consider now the local section

$$
\bar{H}:=\bar{h}\left(\bar{\omega}^{1}\right)^{p-1} \in \bar{M}_{X}^{0}(p-1) .
$$

It is easy to see that the above section does not depend on the choice of $\omega$; hence these local sections are well defined and glue together to give a 'global section' $\bar{H} \in \bar{M}_{X}^{0}(p-1)$ which we may call the Hasse invariant. (This $\bar{H}$ is well known to the experts and is alluded to in [DT94].) Now $\mathcal{L}$ is, of course, algebraizable, i.e. there exists an invertible $\mathcal{O}(X)$-module $L$ such that $\mathcal{L}=L^{\wedge}$. We choose now a section $H \in L^{\otimes(p-1)}$ whose reduction modulo $p$ is $\bar{H}$ and we set

$$
X_{\text {ord }}:=\operatorname{Spec}\left\{\left(\bigoplus_{m \geqslant 0} L^{\otimes m(p-1)}\right) /(H-1)\right\}, \quad S_{\text {ord }}:=\mathcal{O}\left(X_{\text {ord }}\right)^{\wedge} .
$$

If $x$ is a local basis for $L$, then, locally,

$$
X_{\text {ord }}=\operatorname{Spec} \mathcal{O}(X)\left[x^{p-1} / H\right]
$$




\section{A. Buium}

so $X_{\text {ord }}$ is an affine open subscheme of $X$. Note that the $p$-adic completion of $X_{\text {ord }}$ does not depend on the particular choice of $H$. In what follows we let the index 'ord' denote 'base change from $X$ to $X_{\text {ord }}$ '. If $X \otimes k$ contains supersingular points (i.e. false elliptic curves with non-invertible Hasse-Witt matrix), then, of course, $X_{\text {ord }} \otimes k \neq X \otimes k$.

Lemma 4.1. Fix a quaternion algebra $D$. Then, for all except finitely many primes $p$, one can find a Shimura curve $X$ that contains a supersingular $k$-point and a $k$-point corresponding to an ordinary Jacobian.

Proof. Consider a Shimura curve $\mathcal{X}$ over $\mathbb{Z}[1 / N]$ parameterizing false elliptic curves with an appropriate level structure such that the corresponding moduli functor is representable; cf., say, [Buz97]. By [Bui03, Lemma 2.6], for all but finitely many primes $p$, the curve $\mathcal{X} \otimes \boldsymbol{F}_{p}$ has a $k$-point $\left(k=\overline{\boldsymbol{F}}_{p}\right)$ corresponding to an ordinary Jacobian. By [DT94, p. 454, proof of Corollary 3], for all but finitely many primes $p$, the curve $\mathcal{X} \otimes \boldsymbol{F}_{p}$ has a $k$-point corresponding to a supersingular false elliptic curve. We conclude by noting that any two closed points on a curve are contained in an affine open set.

If $X$ is a modular curve then the Hasse invariant is, again, a section $\bar{H} \in \bar{M}_{X}^{0}(p-1)$ and we define $X, X_{\text {ord }}$ by the same formulae as above.

For the rest of this section we continue to assume that $X$ is either a Shimura curve or a modular curve.

We will need later a number of technical facts about the spaces $M_{X}^{r}$ and $M_{X_{\text {ord }}}^{r}$; we collect them in the Corollary 4.3 below. We first prove a general lemma.

Lemma 4.2. Let $B$ be the $p$-adic completion of a smooth $R$-algebra such that $B \otimes k$ is an integral domain. Let $b \in B \backslash p B$ and let $C$ be the $p$-adic completion of $B[1 / b]$. Let

$$
\begin{array}{cl}
J^{r}(\operatorname{Spf} B)=\operatorname{Spf} B^{r}, & J^{r}(\operatorname{Spf} C)=\operatorname{Spf} C^{r}, \\
\bar{B}^{r}=B^{r} \otimes k, & \bar{C}^{r}:=C^{r} \otimes k .
\end{array}
$$

The the following hold:

1) The maps $\bar{B}^{r-1} \rightarrow \bar{B}^{r}$ are injective. In particular the maps $B^{r-1} \rightarrow B^{r}$ are injective with torsion free cokernel. Moreover $\bar{B}^{r}$ and $B^{r}$ are integral domains and we have an equality between groups of units $\left(\bar{B}^{r}\right)^{\times}=\bar{B}^{\times}$.

2) The maps $\bar{B}^{r} \rightarrow \bar{C}^{r}$ are injective. In particular the maps $B^{r} \rightarrow C^{r}$ are injective with torsion free cokernel.

3) $\bar{C}^{r-1} \cap \bar{B}^{r}=\bar{B}^{r-1}$.

4) If $f, g \in B^{r}, f g \in p^{n} B^{r}, f \notin p B^{r}$ then $g \in p^{n} B^{r}$.

5) $B^{r} \cap p^{n} C^{r}=p^{n} B^{r}$.

6) If $c \in\left(C^{r}\right)^{\times}, 1 \leqslant v(p) \leqslant p^{\operatorname{ord}(\bar{v})}$, and $c^{v} \in B^{r+\operatorname{ord}(v)}$, then $c \in B^{r}$. Similarly, if $c \in C^{r}$ and $c^{\phi} \in B^{r+1}$, then $c \in B^{r}$.

Proof. By the 'local product property' of $p$-jet spaces [Bui00, p. 105], Spec $\bar{B}^{r} \rightarrow \operatorname{Spec} \bar{B}^{r-1}$ is a locally trivial bundle in the Zariski topology with fiber an affine space. In particular $\bar{B}^{r}$ are integral domains and $\left(\bar{B}^{r}\right)^{\times}=\left(\bar{B}^{r-1}\right)^{\times}$. Then assertion 1 follows. By the 'compatibility with open immersions' of $p$-jet spaces [Bui00, p. 105], we have $C^{r}=B^{r}[1 / b]^{\wedge}$. So $\bar{C}^{r}=\bar{B}^{r}[1 / \bar{b}]$ is a ring of fractions of $\bar{B}^{r}$; this proves assertion 2 and, due to faithful flatness of $\bar{B}^{r-1} \subset \bar{B}^{r}$, it also proves assertion 3. Now assertions 4 and 5 easily follow by induction. Let us check assertion 6 . We will only check the case $c$ is invertible in $C^{r}$; the case $c$ non-invertible and $v=\phi$ is similar. Let $v=\sum a_{i} \phi^{i}$, $s:=\operatorname{ord}(v), t:=\operatorname{ord}(\bar{v})$. It is enough to prove that for any integer $n \geqslant 1$ we can write

$$
c=b_{n}+p^{n} c_{n}, \quad \text { with } b_{n} \in B^{r}, c_{n} \in C^{r+1} .
$$




\section{Differential MODUlar FORMS ON SHIMURA CURVES, II}

(For if this is so then $b_{n}$ converges to $c$ in $C^{r}$; but $b_{n}$ is a Cauchy sequence in $B^{r}$ due to assertion 5 and $B^{r}$ is complete, so $b_{n}$ converges to an element in $B^{r}$ and we are done.) We shall check (4.3) by induction on $n$. Denote by $x \mapsto \bar{x}$ the canonical surjection $C^{i} \rightarrow \bar{C}^{i}$. To check the case $n=1$ note that since $c^{v} \in B^{r+s}$ we have $c^{v(p)} \in \bar{B}^{r+s}$. Since Spec $B^{r+s}$ is smooth over $k$ and connected, $\bar{B}^{r+s}$ is an integrally closed domain. Also $\bar{B}^{r+s}$ and $\bar{C}^{r+s}$ have the same quotient field. Since $\bar{c}$ is integral over $\bar{B}^{r+s}$ we must have $\bar{c} \in \bar{B}^{r+s}$. By assertion 3 we get $\bar{c} \in \bar{B}^{r}$. So we can write $c=b_{0}+p c_{1}$, $b_{0} \in B^{r}, c_{1} \in C^{r}$; this settles the case $n=1$ in our induction. Assume now that condition (4.3) holds for some $n \geqslant 1$. In particular we must have $b_{n} \in\left(C^{r}\right)^{\times}$so we may write

$$
\begin{aligned}
c^{v} & =\left(b_{n}+p^{n} c_{n}\right)^{a_{0}}\left(b_{n}^{\phi}+p^{n} c_{n}^{\phi}\right)^{a_{1}} \cdots\left(b_{n}^{\phi^{s}}+p^{n} c_{n}^{\phi^{s}}\right)^{a_{s}} \\
& =b_{n}^{v}+p^{n}\left(\sum_{i=0}^{s} a_{i} b_{n}^{v-\phi^{i}} c_{n}^{\phi^{i}}\right)+p^{2 n} \gamma,
\end{aligned}
$$

with $\gamma \in C^{r+s}$. Hence $c^{v}-b_{n}^{v} \in B^{r+s} \cap p^{n} C^{r+s}=p^{n} B^{r+s}$; cf. assertion 5. Write $b_{n}^{v}-c^{v}=p^{n} \beta_{n}$, with $\beta_{n} \in B^{r+s}$. We have $0 \neq \bar{c} \in \bar{C}^{r} \subset \bar{C}^{r+1}, \bar{b}_{n} \neq 0$, and

$$
\bar{\beta}_{n} \bar{b}_{n}^{p^{t}-v(p)}+\sum_{i=0}^{\mathrm{t}} \bar{a}_{i} \bar{b}_{n}^{p^{t}-p^{i}} \bar{c}_{n}^{p^{i}}=0 .
$$

Now the latter equation shows that $\bar{c}_{n}$ is integral over $\bar{B}^{r+s}$. Hence, as before, $\bar{c}_{n} \in \bar{B}^{r+s}$, and hence $\bar{c}_{n} \in \bar{B}^{r}$. So there exists $b \in B^{r}$ and $c_{n+1} \in C^{r}$ such that $c_{n}=b+p c_{n+1}$. Hence $c=b_{n+1}+p^{n+1} c_{n+1}$, where $b_{n+1}=b_{n}+p^{n} b$, and our induction step follows.

Corollary 4.3. The conclusions of Lemma 4.2 are true for

$$
B^{r}=M_{X}^{r}, \quad C^{r}=M_{X_{\text {ord }}}^{r} .
$$

Proof. The conclusions of Lemma 4.2 are local on $B$. But, locally on $X, M_{X_{\text {ord }}}^{r}$ is the $p$-adic completion of $M_{X}^{r}[1 / h]$ for some $h$.

\section{The unit root space}

Let $X$ be either a Shimura curve or a modular curve.

By [Kat73a, p. 178, Theorem 4.1], there exists a subcrystal $U \subset H_{\text {ord }}^{1}$, the unit root subspace, of slope zero, and transversal to $H_{\text {ord }}^{10}$, i.e.

$$
H_{\text {ord }}^{1}=H_{\text {ord }}^{10} \oplus U
$$

The following holds for $X$ a Shimura curve.

Lemma 5.1. The unit root space $U$ is an $\mathcal{O}_{D}$-submodule of $H_{\text {ord }}^{1}$.

Proof. It is enough to check that $U_{P}$ is an $\mathcal{O}_{D}$-submodule of $H_{P}^{1}$ for all $R$-points $P$ of $X_{\text {ord }}$; here the index $P$ means 'base change from $S$ to $R$ via $P$ '. Now, by the functoriality of crystalline cohomology, the semilinear Frobenius $\phi$ acting on the de Rham module $H_{P}^{1}$ commutes with the action of $\mathcal{O}_{D}$. By [Kat73a, p. 172, Theorem 2], there is a basis of $H_{P}^{1}$ of the form $u^{1}, u^{2}, v^{1}, v^{2}$, where $u^{1}, u^{2}$ form a basis of $U_{R}$, and

$$
\begin{gathered}
\phi u=u, \\
\phi v=p B v+C u,
\end{gathered}
$$

where $u:=\left(u^{1}, u^{2}\right)^{\mathrm{t}}, v:=\left(v^{1}, v^{2}\right)^{\mathrm{t}}$, and $B, C \in \operatorname{Mat}_{2}(R)$. Write

$$
i(\alpha)^{*} u=M u+N v, \quad \alpha \in \mathcal{O}_{D}
$$




\section{A. BuIUM}

for some $M, N \in \operatorname{Mat}_{2}(R)$. We get

$$
M u+N v=i(\alpha)^{*}(\phi u)=\phi\left(i(\alpha)^{*} u\right)=M^{\phi} u+N^{\phi}(p B v+C u),
$$

hence $N=p N^{\phi} B$. By induction, $N$ must be divisible by $p^{n}$ for all $n$ so $N=0$. Consequently $i(\alpha)^{*} U \subset U$.

Next we define $\mathcal{U}:=e_{11} U$ in the Shimura curve case and $\mathcal{U}=U$ in the modular curve case. Then $\mathcal{U}$ is a locally free $S_{\text {ord }}$-module of rank one and, clearly, $\mathcal{H}=\mathcal{L} \oplus \mathcal{U}$. We claim that, if $u^{1}$ is a local basis of $\mathcal{U}$ and $\omega=\left(\omega^{1}, \omega^{2}\right)^{\mathrm{t}}$ is an invertible false 1-form or $\omega=\omega^{1}$ is an invertible 1-form (according as $X$ is a Shimura curve or a modular curve), then

$$
\left\langle u^{1}, \omega^{1}\right\rangle_{\theta} \in S_{\text {ord }}^{\times} .
$$

Indeed, if we consider a normalized basis (2.9) or (2.10) respectively and we write

$$
\left(\begin{array}{l}
\omega^{1} \\
u^{1}
\end{array}\right)=\left(\begin{array}{cc}
1 & 0 \\
s_{21} & s_{22}
\end{array}\right)\left(\begin{array}{l}
\omega^{1} \\
\eta^{1}
\end{array}\right)
$$

with $s_{21}, s_{22} \in S_{\text {ord }}$ then $s_{22}$ is invertible and

$$
\left\langle u^{1}, \omega^{1}\right\rangle_{\theta}=s_{22}
$$

which proves our claim.

Finally let us note that if $X$ is a Shimura curve and one is given a basis $u=\left(u^{1}, u^{2}\right)^{\mathrm{t}}$ of $U$ and a false 1-form $\omega=\left(\omega^{1}, \omega^{2}\right)^{\mathrm{t}}$ such that

$$
\left\langle u, \omega^{\mathrm{t}}\right\rangle_{\theta} \in \mathbb{Z}_{p}^{\times} \cdot I
$$

then $e_{11} u^{1}=u^{1}, e_{22} u^{2}=u^{2}$, and $e_{12} u^{1}=d u^{2}$; in particular $u^{1}$ must be a basis of $\mathcal{U}$. Indeed, we may assume the pairing in (5.2) is the identity. Let $\xi^{1}, \xi^{2}$ be the images of $u^{1}, u^{2}$ in $H^{01}$ and set $\xi=\left(\xi^{1}, \xi^{2}\right)^{\mathrm{t}}$. Then

$$
\left\langle\theta_{*}^{-1} \xi, \omega^{\mathrm{t}}\right\rangle=I
$$

So $u=\tau^{U}(\xi)$ where $\tau^{U}: H_{\text {ord }}^{01} \simeq U \subset H_{\text {ord }}^{1}$ is the $\mathcal{O}_{D^{-}}$equivariant splitting defined by $U$ and our claim follows from $(2.8)$.

\section{The forms $f^{\partial}$ and $P$}

Let $X$ be a Shimura curve or a modular curve.

Following [Bar03] let us define the $\delta$-modular form on $X_{\text {ord }}$

$$
f^{\partial} \in M_{X_{\text {ord }}}^{1}(-1+\phi)
$$

as follows. Consider a normalized local basis (2.9) or (2.10) (according as $X$ is a Shimura curve or a modular curve) of $H_{\text {ord }}^{1}$, let $u^{1}$ be a basis of $\mathcal{U}$ and consider the local sections

$$
f^{\partial}:=\frac{\left\langle\phi u^{1}, \omega^{1}\right\rangle_{\theta}}{\phi\left(\left\langle u^{1}, \omega^{1}\right\rangle_{\theta}\right)}\left(\omega^{1}\right)^{\otimes(-1+\phi)} \in M_{X_{\text {ord }}}^{1}(-1+\phi) .
$$

Clearly the above local sections are independent of the choices of $\omega^{1}$ and $u^{1}$ so they glue together to give a global section of $M_{X_{\text {ord }}}^{1}(-1+\phi)$. Similarly, following [Kat73b], we define the $\delta$-modular form on $X_{\text {ord }}$ (which is really 'of order zero', hence 'non-differential')

$$
P \in M_{X_{\text {ord }}}^{0}(2)=\mathcal{L}_{\text {ord }}^{\otimes 2}
$$

as follows. We consider, as before, a normalized local basis (2.9) or (2.10) respectively of $H_{\text {ord }}^{1}$ plus 


\section{Differential modular forms on Shimura Curves, II}

a basis $u^{1}$ of $\mathcal{U}$ and then we consider the local section

$$
P:=\frac{\left\langle u^{1}, \eta^{1}\right\rangle_{\theta}}{\left\langle u^{1}, \omega^{1}\right\rangle_{\theta}} \cdot\left(\omega^{1}\right)^{\otimes 2} \in \mathcal{L}_{\text {ord }}^{\otimes 2}=M_{X_{\text {ord }}}^{0}(2) .
$$

Again this local definition gives a correct global definition.

Let us view $f^{\partial}$ and $H$ as elements of $M_{X_{\text {ord }}}^{1}$ and let us denote by $\bar{f}^{\partial}, \bar{H} \in \bar{M}_{X_{\text {ord }}}^{1}$ their images. We have the following result (due to Barcau [Bar03] in the modular curve case).

Proposition 6.1. $\bar{f}^{\partial}=\bar{H}$ in $\bar{M}_{X_{\text {ord }}}^{1}$.

Proof. Assume $X$ is a Shimura curve; the case of modular curves is similar [Bar03]. Let $\omega$ be a false 1-form, $\xi$ the $\theta$-dual basis of $H_{\text {ord }}^{01}$ and $u$ a basis of the unit root space $U$ such that $\pi(u)=\xi$ where $\pi: H_{\text {ord }}^{1} \rightarrow H_{\text {ord }}^{01}$ is the canonical projection. In particular $\left\langle u, \omega^{\mathrm{t}}\right\rangle_{\theta}=I$, so $u^{1}$ is a basis of $\mathcal{U}$. Write $F^{*} \bar{\xi}=\bar{h} \cdot \bar{\xi}$ as in (4.1). For any $g \in M_{X_{\text {ord }}}^{1}$ let $\bar{g} \in \bar{M}_{X_{\text {ord }}}^{1}$ denote the image of $g$. Also, let $\langle,\rangle_{\bar{\theta}}$ be the pairing on $\bar{H}^{1}:=H^{1} \otimes k$ induced by $\theta$, denote by $v \mapsto \bar{v}$ the natural map $H^{1} \rightarrow \bar{H}^{1}$, and denote by $F^{*}$ the semilinear map on $\bar{H}^{1}$ induced by the p-power Frobenius. We get

$$
\begin{aligned}
\bar{f}^{\partial} & =\left\langle F^{*} \bar{u}^{1}, \bar{\omega}^{1}\right\rangle_{\bar{\theta}}\left(\bar{\omega}^{1}\right)^{\otimes(\phi-1)}=\left\langle\bar{\theta}_{*}^{-1} \pi F^{*} \bar{u}^{1}, \bar{\omega}^{1}\right\rangle\left(\bar{\omega}^{1}\right)^{\otimes(p-1)} \\
& =\left\langle\bar{\theta}_{*}^{-1} F^{*} \pi \bar{u}^{1}, \bar{\omega}^{1}\right\rangle\left(\bar{\omega}^{1}\right)^{\otimes(p-1)}=\left\langle\bar{\theta}_{*}^{-1} F^{*} \bar{\xi}^{1}, \bar{\omega}^{1}\right\rangle\left(\bar{\omega}^{1}\right)^{\otimes(p-1)} \\
& =\bar{h} \cdot\left\langle\bar{\theta}_{*}^{-1} \bar{\xi}^{1}, \bar{\omega}^{1}\right\rangle\left(\bar{\omega}^{1}\right)^{\otimes(p-1)}=\bar{h} \cdot\left(\bar{\omega}^{1}\right)^{\otimes(p-1)}=\bar{H} .
\end{aligned}
$$

Corollary 6.2. There is a form $f_{\partial} \in M_{X_{\text {ord }}}^{1}(1-\phi)$ such that $f_{\partial} \cdot f^{\partial}=1$.

In view of this corollary it is reasonable to define, for any

$$
v=v_{+}+v_{-} \in W, \quad v_{+},-v_{-} \in W_{+},
$$

the form

$$
\left(f^{\partial}\right)^{v}:=\left(f^{\partial}\right)^{v_{+}} \cdot\left(f_{\partial}\right)^{-v_{-}} \in M_{X_{\text {ord }}}^{1}(v(\phi-1)) .
$$

Proof. By Proposition 6.1 it follows that $\bar{f}^{\partial}$ is invertible in $\bar{M}_{X_{\text {ord }}}^{1}$. Since $M_{X_{\text {ord }}}^{1}$ is $p$-adically complete, $f^{\partial}$ itself must be invertible in $M_{X_{\text {ord }}}^{1}$. We may conclude by assertion iv in Proposition 3.1.

\section{Serre-Tate expansion of forms on $X$}

For any weight $w=\sum a_{i} \phi^{i} \in W(r)$ we have a natural ring homomorphism (which we call the Serre-Tate expansion map)

$$
E: M_{X_{\text {ord }}}^{r} \rightarrow S_{\text {for }}^{r}=R[[T]]\left[T^{\prime}, \ldots, T^{(r)}\right]^{\wedge}
$$

defined as follows. First, by functoriality, we have a ring homomorphism

$$
M_{X_{\text {ord }}}^{r} \rightarrow M_{X^{\text {for }}}^{r}
$$

Next assume $X$ is a Shimura curve. The first component $x=x_{\text {for }}:=\omega^{1}$ of the canonical false 1-form $\omega=\omega_{\text {def }, D}$ constructed in [Bui03, $\S 2.4$ ] is a basis of $\mathcal{L}_{\text {for }}$, hence its image $x^{\otimes w}=\omega^{\otimes w}$ in $M_{X \text { for }}^{r}(w)$ is a basis of the latter. (Note that $x$ does not come a priori from a local basis of $\mathcal{L}$ !) Consider the ring homomorphism

$$
M_{X^{\text {for }}}^{r}=R[[T]]\left[x, x^{-1}, x^{\prime}, \ldots, x^{(r)}, T^{\prime}, \ldots, T^{(r)}\right]^{\wedge} \rightarrow S_{\text {for }}^{r}=R[[T]]\left[T^{\prime}, \ldots, T^{(r)}\right]^{\wedge},
$$

defined by $x \mapsto 1, x^{\prime} \mapsto 0, x^{\prime \prime} \mapsto 0$, etc.; note that this homomorphism sends

$$
x^{\otimes w}=x^{a_{0}}\left(x^{\phi}\right)^{a_{1}} \cdots\left(x^{\phi^{r}}\right)^{a_{r}} \mapsto 1,
$$




\section{A. Buium}

hence it maps $M_{X^{\text {for }}}^{r}(w)$ isomorphically onto $S_{\text {for }}^{r}$. We define the map (7.1) as the composition of (7.2) and (7.3). In particular, if we view the map (7.2) as an inclusion, then

$$
f=E(f) x^{\otimes w}
$$

for any $f \in M_{X_{\text {ord }}}^{r}(w)$. If $X$ is a modular curve, the same construction can be performed with $\omega^{1}$ the canonical 1 -form $\omega_{\text {def }}$ in [Bui03, $\left.\S 2.1\right]$.

Now, if $X$ is either a Shimura curve or a modular curve, then, exactly as in [Bui03, Theorem 2.7], we have the following 'Serre-Tate expansion principle'.

THEOREM 7.1. The restriction of the homomorphism (7.1) to each $M_{X_{\text {ord }}}^{r}(w)$ is injective.

Consider now, in the Shimura curve case, the normalized basis $\omega^{1}, \eta^{1}, \omega^{2}, \eta^{2}$ of $H_{\text {for }}^{1}$ whose first vector is $x=\omega^{1}$, cf. (2.9), and let $u=u_{\text {def }, D}$ be the basis of the unit root space $U_{\text {for }}$ defined by Equation (2.6) in [Bui03, $\S 2.1$ ]. In the modular curve case we let $\omega^{1}, \eta^{1}$ be a normalized basis of $H_{\text {for }}^{1}$ with $x=\omega^{1}$ and we let $u=u_{\text {def }}$ be the corresponding basis of $U_{\text {for }}$. So recall that

$$
\phi u=u, \quad\left\langle u, \omega^{\mathrm{t}}\right\rangle=\epsilon \cdot I, \quad \epsilon \in \mathbb{Z}_{p}^{\times}
$$

(cf. [Bui03, $\S \S 2.1$ and 2.2]). (From that work, $\epsilon=1$ in the modular curve case.) In particular $u^{1}$ is a basis for $\mathcal{U}$. Viewing $f^{\partial}$ as an element of $M_{X_{\text {ord }}}^{1}(-1+\phi)$, its image under the Serre-Tate expansion map

$$
E: M_{X_{\text {ord }}}^{1}(-1+\phi) \rightarrow R[[T]]\left[T^{\prime}\right]^{\wedge}
$$

is particularly simple, as the next proposition shows.

Proposition 7.2. $E\left(f^{\partial}\right)=E\left(f_{\partial}\right)=1$. In particular

$$
f^{\partial} \in I_{X_{\text {ord }} \text {,for }}^{1}(\phi-1), \quad f_{\partial} \in I_{X_{\text {ord }} \text {,for }}^{1}(1-\phi) .
$$

Proof. It is enough to prove $E\left(f^{\partial}\right)=1$. Using (7.5), we get that the image of $f^{\partial}$ in $M_{X^{\text {for }}}^{1}$ equals

$$
\frac{\left\langle\phi u^{1}, \omega^{1}\right\rangle}{\phi\left(\left\langle u^{1}, \omega^{1}\right\rangle\right)} x^{-1} x^{\phi}=\frac{\epsilon}{\phi(\epsilon)} x^{-1} x^{\phi}=x^{-1} x^{\phi}
$$

and we are done by (7.4).

In what follows write $u^{1}=s_{21} \omega^{1}+s_{22} \eta^{1}$, with $s_{21} \in R[[T]], s_{22}=R[[T]]^{\times}$, as in (5.1). Note that

$$
\epsilon=\left\langle u^{1}, \omega^{1}\right\rangle=s_{22} \text {. }
$$

Consequently

$$
\left\langle\eta^{1}, u^{1}\right\rangle=s_{21}
$$

hence

and hence

$$
s_{21} x^{2}=-\epsilon \frac{\left\langle u^{1}, \eta^{1}\right\rangle_{\theta}}{\left\langle u^{1}, \omega^{1}\right\rangle_{\theta}} x^{2}=-\epsilon E(P) x^{2},
$$

$$
s_{21}=-\epsilon E(P) \text {. }
$$

By Equations (2.5) and (2.20) in [Bui03, § 2.1], we get

$$
\nabla \omega^{1}=\frac{d T}{1+T} u^{1}=-\epsilon E(P) \frac{d T}{1+T} \omega^{1}+\epsilon \frac{d T}{1+T} \eta^{1} .
$$

In view of Equations (3.3), (3.10), and (3.11) we get

$$
\begin{gathered}
\partial(x)=-E(P) x^{3}, \\
\partial(T)=\epsilon^{-1}(1+T) x^{2} .
\end{gathered}
$$

The next proposition describes the effect of $\partial_{r}$ on Serre-Tate expansions. 


\section{Differential modular forms on Shimura CURVEs, II}

Proposition 7.3. Let $w=\sum_{i-0}^{r} a_{i} \phi^{i}$. Then, for any $f \in M_{X_{\text {ord }}}^{r}(w)$, the following formula holds:

$$
E\left(\partial_{r}(f)\right)=\epsilon^{-1}\left(1+T^{\phi^{r}}\right) \frac{\partial}{\partial T^{(r)}}(E(f))-a_{r} p^{r} E(f)(E(P))^{\phi^{r}} .
$$

Proof. Let $w^{\prime}=w-a_{r} \phi^{r}$. By (7.4) we may write

$$
f=E(f) x^{\otimes w^{\prime}}\left(x^{\phi^{r}}\right)^{a_{r}} .
$$

Then, using Equations (7.6) and (7.7), Proposition 3.1, and Remark 3.2, we may compute:

$$
\begin{aligned}
\partial_{r}(f)= & x^{\otimes w^{\prime}}\left[\partial_{r}(E(f)) \cdot\left(x^{\phi^{r}}\right)^{a_{r}}+E(f) \cdot \partial_{r}\left(\left(x^{\phi^{r}}\right)^{a_{r}}\right)\right] \\
= & x^{\otimes w^{\prime}}\left[\frac{\partial}{\partial T^{(r)}}(E(f)) \partial_{r}\left(T^{(r)}\right) \cdot\left(x^{\phi^{r}}\right)^{a_{r}}+E(f) a_{r}\left(x^{\phi^{r}}\right)^{a_{r}-1} \partial_{r}\left(x^{\phi^{r}}\right)\right] \\
= & x^{\otimes w^{\prime}}\left[\frac{\partial}{\partial T^{(r)}}(E(f)) \epsilon^{-1}\left(1+T^{\phi^{r}}\right)\left(x^{\phi^{r}}\right)^{2}\left(x^{\phi^{r}}\right)^{a_{r}}\right. \\
& \left.+E(f) a_{r}\left(x^{\phi^{r}}\right)^{a_{r}-1}\left(-p^{r} E(P)^{\phi^{r}}\left(x^{\phi^{r}}\right)^{3}\right)\right] \\
= & x^{\otimes w^{\prime}}\left(x^{\phi^{r}}\right)^{a_{r}+2}\left[\frac{\partial}{\partial T^{(r)}}(E(f)) \epsilon^{-1}\left(1+T^{\phi^{r}}\right)-a_{r} p^{r} E(f) E(P)^{\phi^{r}}\right] .
\end{aligned}
$$

This concludes the proof.

Recall from [Bui03, $\S \S 1.8$ and 1.7] respectively that we denoted by $I_{D}(w)$ and $I_{1}(w)$ the spaces of isogeny covariant differential modular forms of weight $w$ in the Shimura curve and the modular curve case respectively; let $I(w)$ be $I_{D}(w)$ or $I_{1}(w)$ according as $X$ is a Shimura or a modular curve. Note that by the Serre-Tate expansion principle in [Bui03, Theorem 2.7], the map

$$
M(w) \otimes_{\mathbb{Z}_{p}} R \rightarrow M_{X}^{r}(w)
$$

in (3.12) is injective; on the other hand, by [Bui03, Theorem 3.12], this map sends $I(w) \otimes_{\mathbb{Z}_{p}} R$ into $I_{X}^{r}(w)$. So we get an injective map

$$
I(w) \otimes_{\mathbb{Z}_{p}} R \rightarrow I_{X}^{r}(w) .
$$

Let $f^{a} \in I\left(-1-\phi^{a}\right)$ be the $\delta$-modular forms defined in [Bui03, $\left.\S 1.10\right]$, and view $f^{1}, f^{\partial}, P^{\phi}$ as elements in $M_{X_{\text {ord }}}^{1}$.

Proposition 7.4. $\partial_{1} f^{1}=f^{\partial}+p P^{\phi} f^{1}$.

Proof. Both members of the equality belong to $M_{X_{\text {ord }}}^{1}(-1+\phi)$; cf. Proposition 3.1. By the SerreTate expansion principle in Theorem 7.1 it is enough to show that the images of the two members of the above equality under

$$
E: M_{X_{\text {ord }}}^{1}(-1+\phi) \rightarrow R[[T]]\left[T^{\prime}\right]^{\wedge}
$$

coincide. Now, by [Bui03, Corollary 2.10] (in the Shimura curve case) and by the proof of [Bui03, Lemma 2.4] (in the modular curve case), $E\left(f^{1}\right)=\epsilon \Psi$ while, by Proposition 7.2 , we have $E\left(f^{\partial}\right)=1$. A trivial computation, using the latter two formulae plus Proposition 7.3, shows that $E\left(\partial_{1} f^{1}\right)$ and $E\left(f^{\partial}+p P^{\phi} f^{1}\right)$ coincide.

Corollary 7.5. $\bar{\partial}_{1} \bar{f}^{1}=\bar{H}$ in $\bar{M}_{X}^{1}$.

Proof. By Propositions 7.4 and 6.1 we have

$$
\bar{\partial}_{1} \bar{f}^{1}=\bar{f}^{\partial}=\bar{H} \in \bar{M}_{X_{\text {ord }}}^{1} .
$$

We conclude by the injectivity of $\bar{M}_{X}^{1} \rightarrow \bar{M}_{X_{\text {ord }}}^{1}$; cf. Corollary 4.3 . 


\section{A. BuIUM}

\section{Proofs of Theorems $1.1,1.2$, and 1.4}

Proof of Theorem 1.4 (Assuming Corollary 1.3). Assertions 1-3 of Theorem 1.4 were proved in [Bui03, Theorem 1.2]. To prove assertion 4 it is enough to show that the $R$-modules

$$
I_{D}(-1-\phi) \otimes_{\mathbb{Z}_{p}} R, \quad I_{D}\left(-1-\phi^{2}\right) \otimes_{\mathbb{Z}_{p}} R, \quad I_{D}\left(-\phi-\phi^{2}\right) \otimes_{\mathbb{Z}_{p}} R
$$

have rank one. Note that

$$
f^{1}, f^{2},\left(f^{1}\right)^{\phi},
$$

are elements of the modules (8.1) respectively; (cf. [Bui03, Corollary 2.10]). So these modules have rank at least one. By Lemma 4.1 one can find $X$ containing a supersingular $k$-point and a point corresponding to an ordinary Jacobian. On the other hand the three modules (8.1) can be embedded into the modules

$$
I_{X}^{1}(-1-\phi), I_{X}^{1}\left(-1-\phi^{2}\right), I_{X}^{1}\left(-\phi-\phi^{2}\right)
$$

via the map (7.8). So the modules (8.1) have rank at most one by Corollary 1.3. This proves assertion 4 in our theorem. Assertion 5 is proved similarly.

Proof of Corollary 1.3 (Assuming Theorem 1.2). The $R$-modules in assertion 1 of the corollary have rank at least one because they contain (the images of the elements) (8.2). The module $I_{X}^{1}(-1-\phi)$ has rank at most one by Theorem 7.1. Hence the module $I_{X}^{1}(-1-\phi)$ has rank one. The modules $I_{X}^{2}\left(-1-\phi^{2}\right)$ and $I_{X}^{2}\left(-\phi-\phi^{2}\right)$ have rank at most 2 by Theorem 7.1 again. However, they cannot have rank 2 due to Theorem 1.2. So they have rank one. This proves assertion 1 in the corollary. Let us prove assertion 2. The case $w(p)<0$ follows directly from Theorem 1.1. So assume

$$
\operatorname{deg}(w)=0, \quad 0<w(p) \leqslant p^{\operatorname{ord}(\bar{w})}-p^{\operatorname{ord}(\bar{w})-1} .
$$

In particular $w \notin p W$. Let us examine the case $w=\phi-1$ first. Take $g \in I_{X}^{1}(\phi-1)$. So, by definition, $E(g)=\lambda \in R$. Consequently the form $h:=g \cdot\left(f^{1}\right)^{\phi}$ belongs to $I_{X}^{2}\left(-1-\phi^{2}\right)$ and has Serre-Tate expansion $E(h)=\lambda \epsilon \Psi^{\phi}$. On the other hand, by [Bui03, Corollary 2.10], the form $f^{2} \in I_{X}^{2}\left(-1-\phi^{2}\right)$ has Serre-Tate expansion

$$
E\left(f^{2}\right)=\epsilon\left(\Psi^{\phi}+p \Psi\right) .
$$

Since, by assertion 1 of Corollary 1.3, $I_{X}^{2}\left(-1-\phi^{2}\right)$ has rank one, it follows that $h$ and $f^{2}$ are linearly dependent; hence $E(h)$ and $E\left(f^{2}\right)$ are linearly dependent. This forces $\lambda=0$ hence $g=0$. This proves assertion 2 for $w=\phi-1$. Let us note that $f^{\partial} \notin M_{X}^{1}$; for if $f^{\partial} \in M_{X}^{1}$, since $f^{\partial} \in M_{X_{\text {ord }}}^{1}(\phi-1)$, it would follow that $f^{\partial} \in M_{X}^{1}(\phi-1)$ (cf. assertion v in Proposition 3.1). Since $E\left(f^{\partial}\right)=1$ we would get $f^{\partial} \in I_{X}^{1}(\phi-1)$, contradicting the case $w=\phi-1$ of assertion 2 that we just proved. Let us prove now assertion 2 for arbitrary $w$ satisfying condition (8.3). Let $g \in I_{X}^{r}(w)$. So again $E(g)=\lambda \in R$. Since $\operatorname{deg}(w)=0$ we may write $w=(\phi-1) v^{\prime}$. Hence

$$
E(g)=E\left(\lambda \cdot\left(f^{\partial}\right)^{v^{\prime}}\right),
$$

hence $g=\lambda \cdot\left(f^{\partial}\right)^{v^{\prime}}$. Note that $m^{\prime}:=\operatorname{ord}\left(\bar{v}^{\prime}\right)=\operatorname{ord}(\bar{w})-1=: m-1$ and $v^{\prime}(p)=w(p) /(p-1)$ so $v^{\prime} \notin p W$ and

$$
1 \leqslant v^{\prime}(p) \leqslant\left(p^{m}-p^{m-1}\right) /(p-1)=p^{m-1}=p^{m^{\prime}} .
$$

If $\lambda \neq 0$ we get

$$
g \in M_{X}^{r} \cap \lambda M_{X}^{r} \text { for }=\lambda M_{X}^{r}
$$

(cf. assertion 5 in Lemma 4.2). So $\left(f^{\partial}\right)^{v^{\prime}} \in M_{X}^{r}$. Since $f^{\partial} \in\left(M_{X_{\text {ord }}}^{1}\right)^{\times}$, by assertion 6 in Lemma 4.2 we get $f^{\partial} \in M_{X}^{1}$, a contradiction. So $\lambda=0$ and, hence, $g=0$. 


\section{Differential modular forms on Shimura Curves, II}

Proof of Theorem 1.2. Assume $\left|\lambda_{v}\right| \leqslant\left|\lambda_{m}\right|$ for all $v$ and seek a contradiction. We may assume

$$
E(g)=\epsilon^{m} p^{n} \Psi^{m}+p^{n} \sum_{v \neq m} \lambda_{v} \Psi^{v}
$$

where $\lambda_{v} \in R$, and the summation extends over all $v \in W_{+}, v \neq m$, such that $\operatorname{ord}(v) \leqslant r-1$ and $\operatorname{deg}(v)=m$. Since $\operatorname{deg}(-w+m(-1-\phi))=0,-w+m(-1-\phi)$ is divisible by $\phi-1$ in $\mathbb{Z}[\phi]$. Write

$$
-w+m(-1-\phi)=-m^{\prime} \cdot(\phi-1), \quad m^{\prime} \in \mathbb{Z}[\phi] .
$$

Similarly, for any $v$ as above, we may write

$$
-w+v(-1-\phi)=-v^{\prime} \cdot(\phi-1), \quad v^{\prime} \in \mathbb{Z}[\phi] .
$$

Note that since $w(p)<-2 m p$ we have $-m^{\prime}(p)>m$. We claim that

$$
g=p^{n}\left(f^{1}\right)^{m}\left(f^{\partial}\right)^{m^{\prime}}+p^{n} \sum_{v \neq m} \lambda_{v}\left(f^{1}\right)^{v}\left(f^{\partial}\right)^{v^{\prime}} \quad \text { in } M_{X_{\text {ord }}}^{r} .
$$

Indeed, both members of Equation (8.4) belong to $M_{X_{\text {ord }}}^{r}(w)$ and, by Proposition 7.2, they have the same image via the Serre-Tate expansion map. By the Serre-Tate expansion principle in Theorem 7.1, Equation (8.4) follows. By Corollary 4.3,

$$
g \in p^{n} M_{X_{\text {ord }}}^{r} \cap M_{X}^{r}=p^{n} M_{X}^{r},
$$

so $g=p^{n} g_{1}$ with $g_{1} \in M_{X}^{r}$ and, hence, by Proposition 6.1,

$$
\bar{g}_{1}=\left(\bar{f}^{1}\right)^{m} \bar{H}^{m^{\prime}(p)}+\sum_{v \neq m} \bar{\lambda}_{v}\left(\bar{f}^{1}\right)^{v(p)} \bar{H}^{v^{\prime}(p)} \quad \text { in } \bar{M}_{X_{\mathrm{ord}}}^{r},
$$

where an upper bar on top of an element in $M_{X_{\text {ord }}}^{r}$ denotes, as usual, the image of the corresponding element in $\bar{M}_{X_{\text {ord }}}^{r}$. By Lemma 4.2, assertion 1, the equality (8.5) holds in $\bar{M}_{X_{\text {ord }}}^{1}$. Now take $\bar{\partial}_{1}^{m}$ in the above equality. By Corollary 7.5, we get

$$
\bar{\partial}_{1}^{m} \bar{g}_{1}=m ! \cdot \bar{H}^{m+m^{\prime}(p)}+\sum_{v \neq m} P(v(p), m) \bar{\lambda}_{v}\left(\bar{f}^{1}\right)^{v(p)-m} \bar{H}^{m+v^{\prime}(p)} \quad \text { in } \bar{M}_{X_{\text {ord }}}^{1},
$$

where we set $P(a, b)=a(a-1) \cdots(a-b+1)$. Since $v(0)<m$ we must have

$$
0=P(v(0), m) \equiv P(v(p), m) \bmod (p),
$$

hence the $\operatorname{sum} \sum_{v \neq m}$ in Equation (8.6) vanishes; so we get

$$
\left(\bar{\partial}_{1}^{m} \bar{g}_{1}\right) \cdot \bar{H}^{-m^{\prime}(p)-m}=\bar{m} ! \neq 0 \quad \text { in } \bar{M}_{X_{\text {ord }}}^{1} .
$$

Since $-m^{\prime}(p)-m>0$, both members of the equality (8.7) belong to $\bar{M}_{X}^{1}$. By Lemma 4.2 , assertion 2, the equality (8.7) holds in $\bar{M}_{X}^{1}$. Hence $\bar{H}$ is invertible in $\bar{M}_{X}^{1}$, hence $\bar{H}$ is invertible in $\bar{M}_{X}^{0}$ (cf. Corollary 4.3). Now if $x$ is a local basis of $\mathcal{L}$, then, locally on $X, \bar{M}_{X}^{0}$ has the form $\bar{S}\left[x, x^{-1}\right]$, hence $\bar{H}$ has, locally on $X$, the form $\bar{h} x^{p-1}$, with $\bar{h} \in \bar{S}^{\times}$. We deduce that $X_{\text {ord }} \otimes k=X \otimes k$, a contradiction. This closes the proof.

Proof of Theorem 1.1. By our assumptions, $\hat{X}=\hat{X}_{\text {ord }}$; we may assume $\Phi=\epsilon^{m} x_{1}^{i_{0}} \cdots x_{r}^{i_{r-1}}$. Set $v=$ $i_{0}+i_{1} \phi+\cdots+i_{r-1} \phi^{r-1}$. Since $\operatorname{deg}(v)=m$ the weight $w+(1+\phi) v$ is divisible by $\phi-1$ in $\mathbb{Z}[\phi]$ so we may write $w+(1+\phi) v=(\phi-1) v^{\prime}$ for some $v^{\prime} \in \mathbb{Z}[\phi]$. But then

$$
E\left(\left(f^{1}\right)^{v} \cdot\left(f^{\partial}\right)^{v^{\prime}}\right)=\epsilon^{m} \cdot \Psi^{v}=\Phi\left(\Psi, \ldots, \phi^{r-1} \Psi\right) .
$$

Let us remark that the proof above shows that, in Theorem 1.1, the set

$$
\left\{\left(f^{1}\right)^{v} \cdot\left(f^{\partial}\right)^{v^{\prime}} \mid v \in W_{+}, \operatorname{ord}(v) \leqslant r, \operatorname{deg}(v)=-\operatorname{deg}(w) / 2\right\}
$$

is a basis of the $K$-linear space $I_{X}^{r}(w) \otimes K$. 


\section{Differential modular forms on Shimura Curves, II}

\section{REFERENCES}

Bar03 M. Barcau, Isogeny covariant differential modular forms and the space of elliptic curves up to isogeny, Compositio Math. 137 (2003), 237-273.

Bui95 A. Buium, Differential characters of Abelian varieties over p-adic fields, Invent. Math. 122 (1995), 309-340.

Bui96 A. Buium, Geometry of p-jets, Duke Math. J. 82 (1996), 349-367.

Bui00 A. Buium, Differential modular forms, J. reine angew. Math. 520 (2000), 95-167.

Bui03 A. Buium, Differential modular forms on Shimura curves, I, Compositio Math. 139 (2003), 197-237.

Buz97 K. Buzzard, Integral models of certain Shimura curves, Duke Math. J. 87 (1997), 591-612.

BZ04 A. Buium and K. Zimmerman, Differential orbit spaces of discrete dynamical systems, Preprint (2004).

DT94 F. Diamond and R. Taylor, Non-optimal levels for mod l modular representations of $G a l(\overline{\mathbb{Q}} / \mathbb{Q})$, Invent. Math. 115 (1994), 435-462.

FC90 G. Faltings and C.-L. Chai, Degeneration of Abelian varieties (Springer, Heidelberg, 1990).

Kat73a N. Katz, Travaux de Dwork, exp. no. 409, Sem. Bourbaki 1971/72, Lecture Notes in Mathematics, vol. 317 (Springer, Berlin, 1973), 167-200.

Kat73b N. Katz, p-adic properties of modular schemes and modular forms, Lecture Notes in Mathematics, vol. 350 (Springer, Berlin, 1973), 69-190.

Alexandru Buium buium@math.unm.edu

Department of Mathematics and Statistics, University of New Mexico, Albuquerque, NM 87131, USA 